\title{
Outcomes of 3309 thoracoabdominal aortic aneurysm repairs
}

\author{
Joseph S. Coselli, MD, ${ }^{\mathrm{a}, \mathrm{d}, \mathrm{e}}$ Scott A. LeMaire, MD, ${ }^{\mathrm{a}, \mathrm{b}, \mathrm{c}, \mathrm{d}, \mathrm{e}}$ Ourania Preventza, MD, ${ }^{\mathrm{a}, \mathrm{d}, \mathrm{e}}$ \\ Kim I. de la Cruz, MD, ${ }^{\mathrm{a}, \mathrm{d}, \mathrm{e}}$ Denton A. Cooley, MD, ${ }^{\mathrm{d}}$ Matt D. Price, MS, ${ }^{\mathrm{a}, \mathrm{d}}$ Alan P. Stolz, MEd, ${ }^{\mathrm{a}, \mathrm{d}}$ \\ Susan Y. Green, MPH, ${ }^{\mathrm{a}, \mathrm{d}}$ Courtney N. Arredondo, MSPH, ${ }^{\mathrm{b}}$ and Todd K. Rosengart, MD ${ }^{\mathrm{a}, \mathrm{c}, \mathrm{d}, \mathrm{e}}$
}

\section{ABSTRACT}

Objective: Since the pioneering era of E. Stanley Crawford, our multimodal strategy for thoracoabdominal aortic aneurysm repair has evolved. We describe our approximately 3-decade single-practice experience regarding 3309 thoracoabdominal aortic aneurysm repairs and identify predictors of early death and other adverse postoperative outcomes.

Methods: We analyzed retrospective (1986-2006) and prospective data (2006-2014) obtained from patients (2043 male; median age, 67 [59-73] years) who underwent 914 Crawford extent I, 1066 extent II, 660 extent III, and 669 extent IV thoracoabdominal aortic aneurysm repairs, of which $723(21.8 \%)$ were urgent or emergency. Repairs were performed to treat degenerative aneurysm $(64.2 \%)$ or aortic dissection $(35.8 \%)$. The outcomes examined included operative death (ie, 30-day or in-hospital death) and permanent stroke, paraplegia, paraparesis, and renal failure necessitating dialysis, as well as adverse event, a composite of these outcomes.

Results: There were 249 operative deaths $(7.5 \%)$. Permanent paraplegia and paraparesis occurred after $97(2.9 \%)$ and $81(2.4 \%)$ repairs, respectively. Of 189 patients $(5.7 \%)$ with permanent renal failure, 107 died in the hospital. Permanent stroke was relatively uncommon $(n=74 ; 2.2 \%)$. The rate of the composite adverse event $(n=478 ; 14.4 \%)$ was highest after extent II repair $(\mathrm{n}=203 ; 19.0 \%)$ and lowest after extent IV repair $(\mathrm{n}=67 ; 10.2 \% ; P<.0001)$. Estimated postoperative survival was $83.5 \% \pm 0.7 \%$ at 1 year, $63.6 \% \pm 0.9 \%$ at 5 years, $36.8 \% \pm 1.0 \%$ at 10 years, and $18.3 \% \pm 0.9 \%$ at 15 years.

Conclusions: Repairing thoracoabdominal aortic aneurysms poses substantial risks, particularly when the entire thoracoabdominal aorta (extent II) is replaced. Nonetheless, our data suggest that thoracoabdominal aortic aneurysm repair, when performed at an experienced center, can produce respectable outcomes. (J Thorac Cardiovasc Surg 2016;151:1323-38)

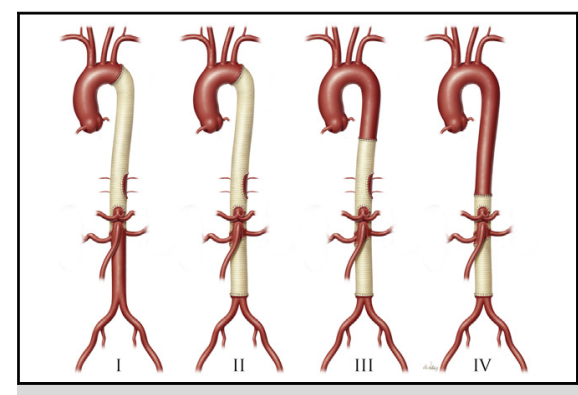

Outcomes of TAAA repair differ by Crawford extent.

Central Message

Open TAAA repair produces respectable outcomes, but there is clearly room for improvement. Outcome differs by repair extent.

\section{Perspective}

We present the results of 3309 open TAAA repairs to elucidate operative risk. These repairs require interrupting blood flow to vital organs, which incurs the risk of postoperative paraplegia, renal failure, and other complications. Our data suggest that open TAAA repair performed at an experienced center can produce respectable outcomes, but further improvement is needed.

See Editorial Commentary page 1339.

See Editorial page 1232.
Thoracoabdominal aortic aneurysm (TAAA) repair was first performed in the $1950 \mathrm{~s}^{1-3}$ and soon became a signature repair under Michael E. DeBakey's tutelage at Baylor

\footnotetext{
From the a Division of Cardiothoracic Surgery, ${ }^{\mathrm{b}}$ Surgical Research Core, Michael E. DeBakey Department of Surgery, ${ }^{\mathrm{c}}$ Cardiovascular Research Institute, Baylor College of Medicine, Houston, Tex; ${ }^{\mathrm{d}}$ Department of Cardiovascular Surgery, Texas Heart Institute, Houston, Tex; and ${ }^{\mathrm{e}} \mathrm{CHI}$ St Luke's Health-Baylor St Luke's Medical Center, Houston, Tex.

Read at the 95th Annual Meeting of The American Association for Thoracic Surgery, Seattle, Washington, April 25-29, 2015.

Received for publication May 1, 2015; revisions received Dec 1, 2015; accepted for publication Dec 14, 2015; available ahead of print Feb 19, 2016.

Address for reprints: Joseph S. Coselli, MD, One Baylor Plaza, BCM 390, Houston, TX 77030 (E-mail: jcoselli@bcm.edu).

$0022-5223 / \$ 36.00$

Copyright $(2016$ by The American Association for Thoracic Surgery http://dx.doi.org/10.1016/j.jtcvs.2015.12.050
}

College of Medicine ${ }^{4}$ - the premier aortic center of this pioneering era. Into the 1970 s and beyond, E. Stanley Crawford refined TAAA repair by invoking many surgical concepts that remain in use today: endoaortic graft inclusion, expeditious repair, reattachment of intercostal and lumbar arteries, and patch reattachment of visceral vessels, to name a few. ${ }^{5}$ Through the efforts of Drs DeBakey, Denton A. Cooley, and Crawford and their

Scanning this $\mathrm{QR}$ code will take you to the article title page. To view the AATS 2015 Webcast, see the URL at the end of the article.

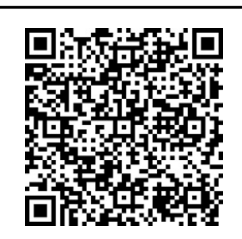




\section{Abbreviations and Acronyms \\ eTAAR = endovascular thoracoabdominal aortic repair \\ $\mathrm{HCA}=$ hypothermic circulatory arrest \\ MOF = multisystem organ failure \\ $\mathrm{RRR}=$ relative risk ratio \\ TAAA $=$ thoracoabdominal aortic aneurysm \\ TEVAR $=$ thoracic endovascular aortic repair}

colleagues, Baylor College of Medicine developed into a global, tertiary-care aortic center that continues to treat many patients with highly complex TAAA pathology who are in acute need. Even after his untimely death, Crawford continued to guide TAAA repair through the many surgeons he had mentored, the widespread use of his namesake classification system (the Crawford extents of TAAA repair $^{6}$ ) (Figure 1), and information gleaned from his published experience of 1509 TAAA repairs. ${ }^{7}$ As we assess postoperative outcomes during our approximately 3-decade single-practice experience regarding 3309 TAAA repairs, we honor the legendary men that laid the foundation of modern TAAA repair.
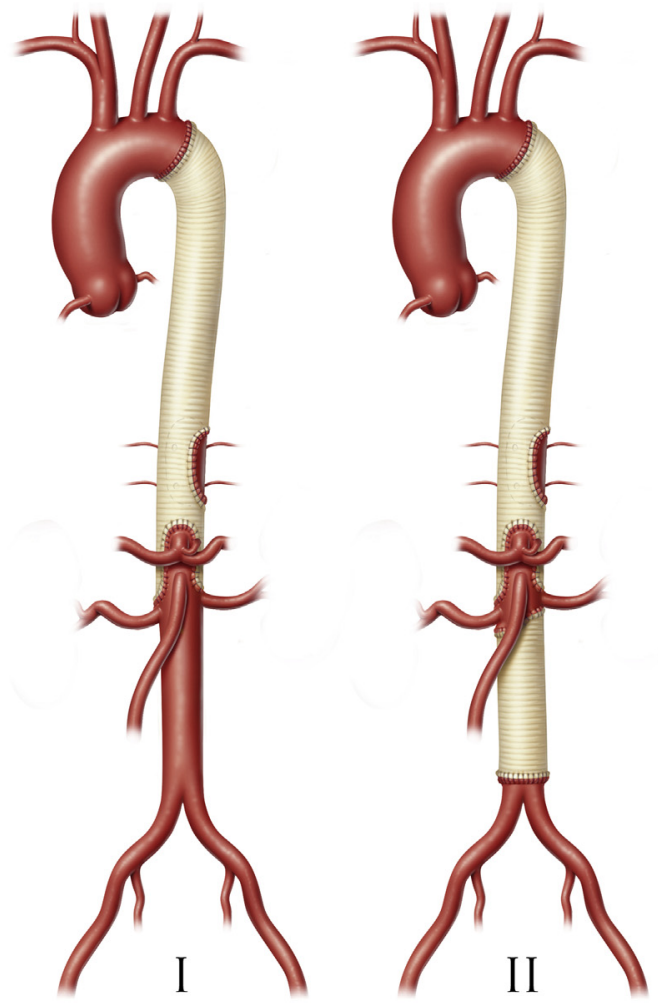

\section{PATIENTS AND METHODS \\ Study Enrollment and Patient Characteristics}

Baylor College of Medicine's institutional review board approved our clinical research protocol in 2006. For patients who underwent surgery after protocol approval, data were collected prospectively by dedicated staff, and informed consent was obtained whenever possible; waiver of consent was approved for patients who could not provide consent because of illness and whose family members were not available. For patients who underwent surgery before protocol approval, data were collected retrospectively from medical records, and consent was waived.

From October 1986 to December 2014, 3320 consecutive open TAAA repairs were performed on our service. We were unable to obtain consent from 11 patients with repairs performed after our protocol was approved; these patients were excluded from our analyses, and the remaining 3309 repairs form the basis of this report (Figure 2). Repairs included 914 Crawford extent I TAAA repairs, 1066 extent II repairs, 660 extent III repairs, and 669 extent IV repairs (Table 1). Of these, 142 repairs $(4.3 \%)$ involved patients living outside the United States, in 28 different countries. Patients were characterized as symptomatic $(n=2117 ; 64.0 \%)$ if they had any symptom related to their aortic disease (eg, pain, hoarseness, dysphagia); in 729 (21.8\%), repair was nonelective, with patients presenting in acute distress or being transferred to us for urgent or emergency care. Approximately two thirds of repairs were performed to treat degenerative aneurysms without dissection $(64.2 \%)$; the other one third were performed to treat aortic dissection $(35.8 \%)$, including the late aortic dilatation that is common in chronic dissection $(30.8 \%)$. The distributions of several preoperative characteristics differed significantly by Crawford extent of repair (Table 1), including age, aortic dissection,
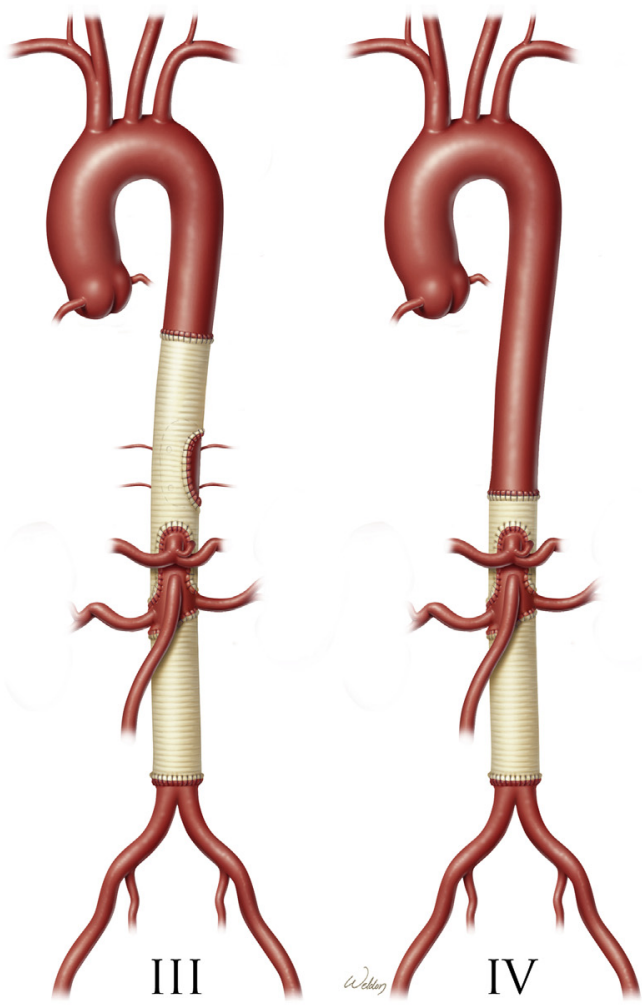

FIGURE 1. Drawing illustrating the Crawford extents of thoracoabdominal aortic aneurysm (TAAA) repair. Extent I repairs involve most or all of the descending thoracic aorta and the upper abdominal aorta. Extent II repairs involve the same segments as extent I repairs but also extend into the infrarenal abdominal aorta. Extent III repairs involve a combination of the distal half, or less, of the descending thoracic aorta (beginning below the sixth rib) and varying portions of the abdominal aorta. Extent IV repairs involve the abdominal aorta below the diaphragm. Used with permission of Baylor College of Medicine. 


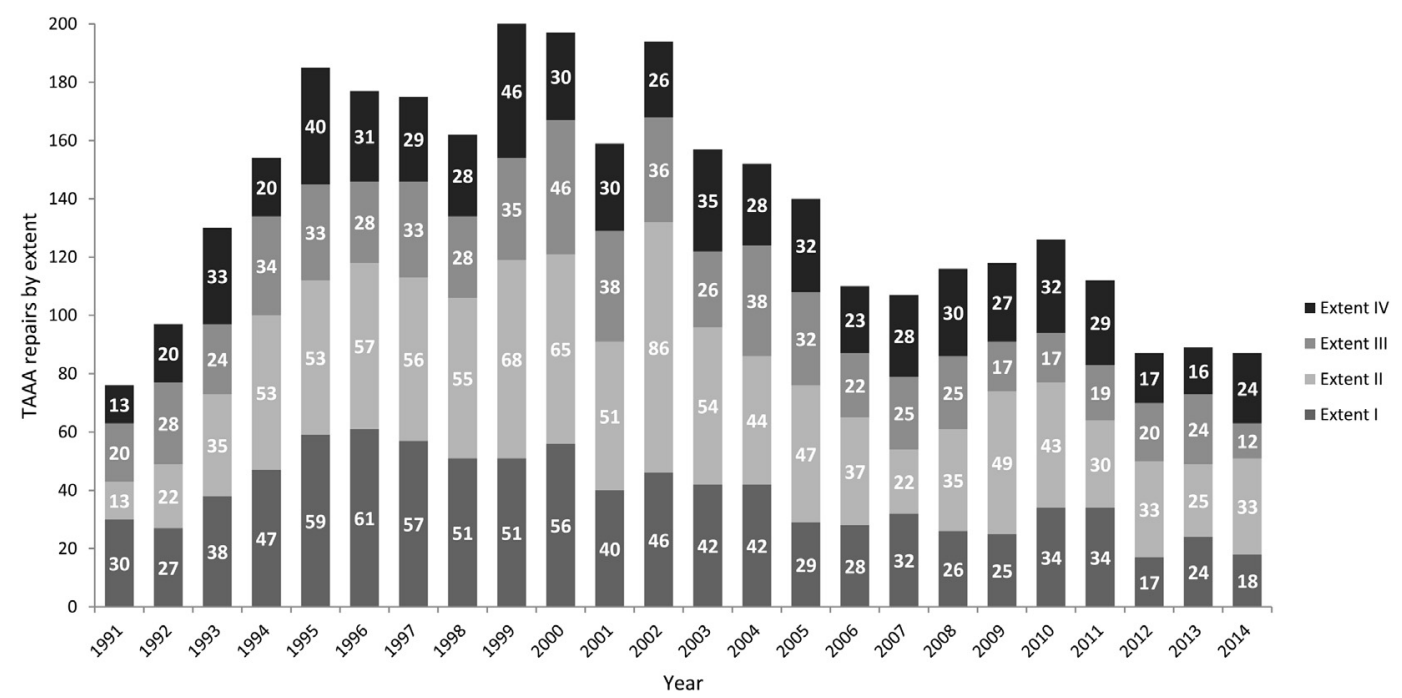

FIGURE 2. Graph depicting the number of TAAA repairs, stratified by Crawford extent, performed between January 1991 and December 2014. Two repairs, 1 performed in October 1986 and the other in April 1990, are not shown. TAAA, Thoracoabdominal aortic aneurysm.

coronary artery disease, cerebrovascular disease, serum creatinine, pulmonary disease, and peripheral vascular disease (all with $P<.001$ ).

\section{Study Definitions and Follow-up}

Operative mortality was defined as death within 30 days of surgery or before final discharge from hospitalization (including acute care at other facilities). ${ }^{8}$ Adverse event, a composite end point, was defined as operative death or permanent stroke, paraplegia, paraparesis, or renal failure necessitating dialysis. ${ }^{9}$ Complications were considered permanent if they were present at the time of hospital discharge or early death. Survivors were defined as having a life-altering complication if they were discharged with stroke, paraplegia, paraparesis, or renal failure necessitating dialysis. Preoperative, operative, and outcomes data were collected according to standardized definitions reported in our recent publications. ${ }^{10,11}$ Patients with lower-extremity neurologic deficits not due to stroke were considered to have spinal cord deficits, namely, paraplegia or paraparesis. Visceral artery procedure was defined as endarterectomy, stenting, or bypass of visceral arteries. Postoperative complications were captured regardless of whether they were immediate or delayed, including delayed complications related to multisystem organ failure (MOF). We categorized MOF as primary MOF when several organ systems failed simultaneously because of the physiologic insult of the operation or as secondary MOF when organ systems failed progressively after a specific inciting complication, such as stroke or pneumonia. Postoperative repair failure was defined as failure directly involving the index TAAA graft, namely, pseudoaneurysm, patch aneurysm, fistula, or graft infection, and did not include subsequent repair necessitated by progression of aortic disease adjacent to the repair. ${ }^{12}$

Follow-up data were obtained by clinic visit, telephone, or written correspondence and were available for all but 34 patients $(1.0 \%)$, who were lost at hospital discharge and remained without 30-day follow-up. Medical records and Social Security Death Index data were reviewed for late events. Recent follow-up data (from September 2013 or later) were available for $1101(92.1 \%)$ of 1196 late survivors, including 1048 $(96.3 \%)$ of surviving US patients. Fifty-five international patients $(1.7 \%)$ lacked recent follow-up data (20 were lost at hospital discharge $<30$ days postoperatively) and were assumed to be alive; 40 US patients $(1.2 \%)$ lacked recent follow-up data (14 were lost at hospital discharge $<30$ days postoperatively) and were indicated to be alive by the Social Security Death Index. The median follow-up time was 6.03 [2.9-11.1] years (range, 30 days to 23.1 years) for surviving patients with at least 30-day follow-up after repair $(\mathrm{n}=1162)$ and 6.2 [3.1-11.5] years (range, 33 days to 23.1 years) for US patients with recent follow-up $(\mathrm{n}=1048)$.

\section{Surgical Techniques}

Our technique for open repair of TAAA has evolved over the last 3 decades into a multimodal approach that is based on the Crawford extents of repair and other selective factors (eg, prior distal aortic repair, poor cardiac function, other health factors). By 1998, all of the varied techniques (Table 2) that form our current operative approach (described in detail previously $^{13-15}$ and illustrated in a companion article ${ }^{16}$ ) had been introduced to our clinical practice, but their use had not yet been standardized, and several techniques were in their infancy (Era 1, $\mathrm{n}=1158$ ). As we shifted into an investigational period (1999-2004; Era $2, \mathrm{n}=1059$ ), we retrospectively reviewed our clinical data regarding the use of left heart bypass, ${ }^{17}$ conducted randomized clinical trials on the use of cerebrospinal fluid drainage ${ }^{18}$ and cold renal perfusion, ${ }^{19}$ and described our experience with using balloon-expandable stents to manage visceral artery lesions. ${ }^{20}$ In 2005, we published our multimodal approach to TAAA repair, ${ }^{21}$ which largely standardized our approach to repair in the contemporary era (2005-2014; Era 3, n = 1092); however, we continued to evaluate innovative approaches, and in 2009, we published our second randomized clinical trial evaluating different approaches to cold renal perfusion. $^{22}$

In brief, for all extents of repair, we routinely use moderate systemic heparinization $(1.0 \mathrm{mg} / \mathrm{kg})$ and mild permissive hypothermia $\left(32^{\circ} \mathrm{C}-34^{\circ} \mathrm{C}\right.$, nasopharyngeal); when possible, we reattach 1 or more pairs of intercostal and lumbar arteries (especially between T8 and L1) and intermittently perfuse the renal arteries with a cold $\left(4^{\circ} \mathrm{C}\right)$ solution. Operative adjuncts that are generally reserved for more extensive TAAA repairs (ie, Crawford extents I and II) include cerebrospinal fluid drainage, left heart bypass, and selective perfusion of the celiac and superior mesenteric arteries. As needed, visceral arteries are managed with endarterectomy, balloon-expandable stents, or bypass grafts. To treat dissection extending into the visceral arteries, the septum is fenestrated, excised, sutured closed, or obliterated with a small balloon-expandable stent. When the visceral arteries are especially fragile or significantly displaced, 1 or more branch-grafts are used. ${ }^{15}$ We rarely use deep hypothermic circulatory arrest (HCA), usually only because safe proximal 
TABLE 1. Preoperative characteristics

\begin{tabular}{|c|c|c|c|c|c|c|}
\hline Variable & $\begin{array}{c}\text { All } \\
\mathbf{n}=\mathbf{3 3 0 9}\end{array}$ & $\begin{array}{l}\text { Extent I } \\
n=914\end{array}$ & $\begin{array}{l}\text { Extent II } \\
\mathrm{n}=\mathbf{1 0 6 6}\end{array}$ & $\begin{array}{c}\text { Extent III } \\
n=660\end{array}$ & $\begin{array}{c}\text { Extent IV } \\
n=669\end{array}$ & $P$ value \\
\hline Age (y) & 67 [59-73] & $67[57-73]$ & $66[56-72]$ & 69 [61-74] & $70[64-75]$ & $<.001$ \\
\hline Age $\leq 50 \mathrm{y}$ & $439(13.3)$ & $131(14.3)$ & $192(18.0)$ & $69(10.5)$ & $47(7.0)$ & $<.001$ \\
\hline Age $>79 y$ & $193(5.8)$ & $38(4.2)$ & $38(3.6)$ & $56(8.5)$ & $61(9.1)$ & $<.001$ \\
\hline Male & $2043(61.7)$ & $513(56.1)$ & $691(64.8)$ & $378(57.3)$ & $461(68.9)$ & $<.001$ \\
\hline Genetically triggered disorder & $523(15.8)$ & $153(16.7)$ & $227(21.3)$ & $87(13.2)$ & $56(8.4)$ & $<.001$ \\
\hline Connective tissue disorder & $330(10.0)$ & $67(7.3)$ & $177(16.6)$ & $56(8.5)$ & $30(4.5)$ & $<.001$ \\
\hline Marfan syndrome & $288(8.7)$ & $53(5.8)$ & $154(14.5)$ & $52(7.9)$ & $29(4.3)$ & $<.001$ \\
\hline Aortic aneurysm without dissection & $2124(64.2)$ & $484(53.0)$ & $538(50.5)$ & $519(78.6)$ & $583(87.1)$ & $<.001$ \\
\hline Aortic dissection & $1185(35.8)$ & $430(47.1)$ & $528(49.5)$ & $141(21.4)$ & $86(12.9)$ & $<.001$ \\
\hline Acute dissection & $110(3.3)$ & $47(5.1)$ & $41(3.9)$ & $13(2.0)$ & $9(1.4)$ & $<.001$ \\
\hline Subacute dissection & $55(1.7)$ & $31(3.4)$ & $18(1.7)$ & $4(0.6)$ & $2(0.3)$ & $<.001$ \\
\hline Acute or subacute dissection & $165(5.0)$ & $78(8.5)$ & $59(5.5)$ & $17(2.6)$ & $11(1.6)$ & $<.001$ \\
\hline Chronic dissection & $1020(30.8)$ & $352(38.5)$ & $469(44.0)$ & $124(18.8)$ & $75(11.2)$ & $<.001$ \\
\hline DeBakey type I & $427(12.9)$ & $150(16.4)$ & $216(20.3)$ & $32(4.9)$ & $29(4.3)$ & $<.001$ \\
\hline DeBakey type IIIa & $121(3.7)$ & $59(6.5)$ & $33(3.1)$ & $17(2.6)$ & $12(1.8)$ & $<.001$ \\
\hline DeBakey type IIIb & $607(18.3)$ & $219(24.0)$ & $271(25.4)$ & $78(11.8)$ & $39(5.8)$ & $<.001$ \\
\hline Localized dissection & $33(1.0)$ & $4(0.4)$ & $9(0.8)$ & $14(2.1)$ & $6(0.9)$ & .009 \\
\hline Maximum distal aortic diameter, $\mathrm{cm}$ & $\begin{array}{l}6.2[5.5-7.2] \\
(\mathrm{n}=2936)\end{array}$ & $\begin{array}{c}6.2[5.5-7.2] \\
(\mathrm{n}=801)\end{array}$ & $\begin{array}{c}6.3[5.7-7.2] \\
(\mathrm{n}=958)\end{array}$ & $\begin{array}{c}6.2[5.6-7.2] \\
(\mathrm{n}=586)\end{array}$ & $\begin{array}{c}6.1[5.4-7.2] \\
(\mathrm{n}=591)\end{array}$ & .02 \\
\hline Hypertension & $2805(84.8)$ & $765(83.7)$ & $917(86.0)$ & $555(84.1)$ & $568(84.9)$ & .5 \\
\hline Hyperlipidemia & $967(29.2)$ & $229(25.1)$ & $263(24.7)$ & $215(32.6)$ & $260(38.9)$ & $<.001$ \\
\hline Diabetes & $261(7.9)$ & $63(6.9)$ & $67(6.3)$ & $56(8.5)$ & $75(11.2)$ & .001 \\
\hline Coronary artery disease & $1232(37.2)$ & $259(28.3)$ & $351(32.9)$ & $291(44.1)$ & $331(49.5)$ & $<.001$ \\
\hline Previous CABG & $496(15.0)$ & $98(10.7)$ & $136(12.8)$ & $121(18.3)$ & $141(21.1)$ & $<.001$ \\
\hline Prior myocardial infarction & $652(19.7)$ & $131(14.3)$ & $181(17.0)$ & $162(24.6)$ & $178(26.6)$ & $<.001$ \\
\hline History of atrial arrhythmia & $402(12.2)$ & 125 (13.7) & $119(11.2)$ & $81(12.3)$ & $77(11.5)$ & .4 \\
\hline History of LV hypertrophy & $1236(37.4)$ & $330(36.1)$ & $410(38.5)$ & $258(39.1)$ & $238(35.6)$ & .4 \\
\hline Cerebrovascular disease & 575 (17.4) & $117(12.8)$ & $183(17.2)$ & $144(21.8)$ & $131(19.6)$ & $<.001$ \\
\hline Prior stroke & $334(10.1)$ & $67(7.3)$ & $113(10.6)$ & $82(12.4)$ & $72(10.8)$ & .007 \\
\hline Prior transient ischemic attack & $158(4.8)$ & $40(4.4)$ & $49(4.6)$ & $38(5.8)$ & $31(4.6)$ & .6 \\
\hline Chronic renal insufficiency* & $99(3.0)$ & $15(1.6)$ & $28(2.6)$ & $30(4.6)$ & $26(3.9)$ & .004 \\
\hline Chronic renal failure (dialysis) & $60(1.8)$ & $11(1.2)$ & $17(1.6)$ & $18(2.7)$ & $14(2.1)$ & .1 \\
\hline Preoperative serum creatinine level, $\mathrm{mg} / \mathrm{dL}$ & $\begin{array}{c}1.1[0.9-1.4] \\
(\mathrm{n}=3037)\end{array}$ & $\begin{array}{c}1.05[0.9-1.3] \\
\quad(\mathrm{n}=822)\end{array}$ & $\begin{array}{c}1.1[0.9-1.4] \\
(\mathrm{n}=980)\end{array}$ & $\begin{array}{c}1.2[0.9-1.5] \\
(\mathrm{n}=614)\end{array}$ & $\begin{array}{c}1.16[0.9-1.5] \\
\quad(\mathrm{n}=621)\end{array}$ & $<.001$ \\
\hline Atrophic kidney & $325(9.8)$ & $43(4.7)$ & $108(10.1)$ & $82(12.4)$ & $92(13.8)$ & $<.001$ \\
\hline Polycystic kidney disease & $211(6.4)$ & $52(5.7)$ & $67(6.3)$ & $45(6.8)$ & $47(7.0)$ & .7 \\
\hline Previous nephrectomy (whole) & $74(2.2)$ & $15(1.6)$ & $26(2.4)$ & $19(2.9)$ & $14(2.1)$ & .4 \\
\hline Pulmonary disease & $1298(39.2)$ & $314(34.4)$ & $363(34.1)$ & $322(48.8)$ & 299 (44.7) & $<.001$ \\
\hline COPD & $1214(36.7)$ & $288(31.5)$ & $334(31.3)$ & $303(45.9)$ & $289(43.2)$ & $<.001$ \\
\hline Past or current tobacco use & $2616(79.1)$ & $669(73.2)$ & $835(78.3)$ & $546(82.7)$ & $566(84.6)$ & $<.001$ \\
\hline Asymptomatic & $1192(36.0)$ & $311(34.0)$ & $400(37.5)$ & $239(36.2)$ & $242(36.2)$ & .5 \\
\hline Symptomatic & $2117(64.0)$ & $603(66.0)$ & $666(62.5)$ & $421(63.8)$ & $427(63.8)$ & .5 \\
\hline Acute symptoms & $549(16.6)$ & $169(18.5)$ & $161(15.1)$ & $109(16.5)$ & $110(16.4)$ & .3 \\
\hline Chronic symptoms & $1664(50.3)$ & $465(50.9)$ & $538(50.5)$ & $328(49.7)$ & $333(49.8)$ & 1.0 \\
\hline Peripheral vascular disease & $855(25.8)$ & $148(16.2)$ & $252(23.6)$ & $191(28.9)$ & $264(39.5)$ & $<.001$ \\
\hline Rupture & $170(5.1)$ & $48(5.3)$ & $32(3.0)$ & $43(6.5)$ & $47(7.0)$ & .001 \\
\hline Prior distal aortic repair $\dagger$ & $858(25.9)$ & 143 (15.6) & $197(18.5)$ & 275 (41.7) & $243(36.3)$ & $<.001$ \\
\hline Failure of prior aortic repair & $136(4.1)$ & $9(1.0)$ & $23(2.2)$ & $47(7.1)$ & $57(8.5)$ & $<.001$ \\
\hline
\end{tabular}

Values are $\mathrm{n}(\%)$ or median [interquartile range]. $C A B G$, Coronary artery bypass graft; $L V$, left ventricular; $C O P D$, chronic obstructive pulmonary disease. *Serum creatinine level $\geq 3.0 \mathrm{mg} / \mathrm{dL}$ or dialysis. $\dagger$ Prior open or endovascular repair. $\ddagger$ Failure of prior distal open or endovascular repair necessitating the in-window repair.

aortic clamping is precluded by frank rupture, enormous aneurysm, or other complicating factors. During extent II, III, and IV repairs, the distal anastomosis usually is constructed at the level of the aortic bifurcation (or, occasionally, to each iliac or femoral artery separately).

\section{Data Presentation}

Data were analyzed with IBM SPSS Statistics 22 (International Business Machines Corp, Armonk, NY) and Stata IC 13 (StataCorp 2013. Stata Statistical Software: Release 13. StataCorp LP, College Station, 
TABLE 2. Operative details

\begin{tabular}{|c|c|c|c|c|c|c|}
\hline Variable & $\begin{array}{c}\text { All } \\
\mathbf{n}=\mathbf{3 3 0 9}\end{array}$ & $\begin{array}{l}\text { Extent I } \\
n=914\end{array}$ & $\begin{array}{l}\text { Extent II } \\
n=1066\end{array}$ & $\begin{array}{c}\text { Extent III } \\
\mathbf{n}=660\end{array}$ & $\begin{array}{c}\text { Extent IV } \\
\mathbf{n}=669\end{array}$ & $P$ value \\
\hline \multicolumn{7}{|l|}{ Urgency of operation } \\
\hline Elective & $2586(78.2)$ & $700(76.6)$ & $866(81.2)$ & $504(76.4)$ & $516(77.1)$ & .03 \\
\hline Urgent & $428(12.9)$ & $131(14.3)$ & $127(11.9)$ & $86(13.0)$ & $84(12.6)$ & .4 \\
\hline Emergency & $295(8.9)$ & $83(9.1)$ & $73(6.8)$ & $70(10.6)$ & $69(10.3)$ & .02 \\
\hline Urgent or emergency & $723(21.8)$ & $214(23.4)$ & $200(18.8)$ & $156(23.6)$ & $153(22.9)$ & .03 \\
\hline \multicolumn{7}{|l|}{ Aortic repair details } \\
\hline Redo thoracotomy & $563(17.0)$ & $84(9.2)$ & $125(11.7)$ & $192(29.1)$ & $162(24.4)$ & $<.001$ \\
\hline Extraction of endograft* & $48(1.5)$ & $9(1.0)$ & $13(1.2)$ & $9(1.4)$ & $17(2.5)$ & .06 \\
\hline Extraction of TEVAR & $29(0.9)$ & $8(0.9)$ & $12(1.1)$ & $6(0.9)$ & $3(0.4)$ & .5 \\
\hline Extraction of EVAR & $24(0.7)$ & $1(0.1)$ & $3(0.3)$ & $5(0.8)$ & $15(2.2)$ & $<.001$ \\
\hline Reverse elephant trunk & $80(2.4)$ & $32(3.5)$ & $48(4.5)$ & 0 & 0 & $<.001$ \\
\hline Elephant trunk completion repair & $133(4.0)$ & $60(6.6)$ & $73(6.8)$ & 0 & 0 & $<.001$ \\
\hline Clamping proximal to LSCA & $522(15.8)$ & $272(29.8)$ & $245(23.0)$ & $2(0.3)$ & $3(0.4)$ & $<.001$ \\
\hline ICA/LA reattachment & $1675(50.6)$ & $498(54.5)$ & $941(88.3)$ & $221(33.5)$ & $15(2.2)$ & $<.001$ \\
\hline Iliac/femoral bypass graft & $270(8.2)$ & $2(0.2)$ & $95(8.9)$ & $59(8.9)$ & $114(17.0)$ & $<.001$ \\
\hline Total estimated blood loss, $\mathrm{mL}$ & $2500[1500-5000]$ & $2500[1500-4500]$ & 3400 [2000-6500] & $2000[1000-4000]$ & 2000 [1000-3900] & $<.001$ \\
\hline Splenectomy & $401(12.1)$ & $96(10.5)$ & $115(10.8)$ & $95(14.4)$ & $95(14.2)$ & .02 \\
\hline Cholecystectomy & $72(2.2)$ & $8(0.9)$ & $25(2.3)$ & $22(3.3)$ & $17(2.5)$ & .007 \\
\hline \multicolumn{7}{|l|}{ Clamp and ischemic times } \\
\hline Aortic clamp time, min & $48[36-62]$ & $41[32-52]$ & $63[53-75]$ & $43[34-53]$ & $41[31-51]$ & $<.001$ \\
\hline Intercostal, total, min & $40[30-52]$ & $40[31-50]$ & $52[41-64]$ & $35[27-44]$ & $31[24-40]$ & $<.001$ \\
\hline Intercostal, unprotected, min & 31 [24-39] & $26[20-32]$ & 35 [27-42] & 34 [26-42] & $31[24-40]$ & $<.001$ \\
\hline Left renal, total, min & $45[33-59]$ & $40[31-50]$ & $60[50-73]$ & $39[30-50]$ & $35[27-47]$ & $<.001$ \\
\hline Left renal, unprotected, min & $35[27-46]$ & 27 [20-33] & $42[35-52]$ & 38 [29-48] & 35 [26-47] & $<.001$ \\
\hline Right renal, total, min & $42[32-56]$ & $40[31-50]$ & 57 [48-69] & 37 [29-45] & $32[25-42]$ & $<.001$ \\
\hline Right renal, unprotected, min & $33[26-42]$ & 27 [20-33] & 40 [33-47] & 35 [29-44] & $32[25-42]$ & $<.001$ \\
\hline Celiac axis, total, min & $42[22-55]$ & $40[31-50]$ & 57 [48-69] & 37 [29-46] & $32[25-41]$ & $<.001$ \\
\hline Celiac axis, unprotected, min & 33 [26-42] & $27[20-33]$ & $39[32-46]$ & $36[29-45]$ & $32[25-41]$ & $<.001$ \\
\hline SMA, total, min & $42[32-55]$ & $40[31-50]$ & $57[48-68]$ & $37[29-46]$ & $32[25-41]$ & $<.001$ \\
\hline SMA, unprotected, min & $33[26-42]$ & $27[20-33]$ & $39[32-47]$ & $36[29-44]$ & $32[25-41]$ & $<.001$ \\
\hline Left leg, total, min & $46[35-59]$ & $40[31-50]$ & $62[52-73]$ & $41[32-50]$ & $39[31-48]$ & $<.001$ \\
\hline Left leg, unprotected, min & 37 [28-47] & $27[20-33]$ & $44[36-52]$ & 39 [31-49] & $39[31-48]$ & $<.001$ \\
\hline Right leg, total, min & $46[35-59]$ & $40[31-50]$ & $61[52-72]$ & $41[32-50]$ & $40[31-49]$ & $<.001$ \\
\hline Right leg, unprotected, min & 37 [28-47] & $27[20-33]$ & $44[36-52]$ & 39 [31-49] & $39[31-48]$ & $<.001$ \\
\hline \multicolumn{7}{|l|}{ Management of visceral/renal arteries } \\
\hline Bypass graft & $825(24.9)$ & $38(4.2)$ & $334(31.3)$ & $214(32.4)$ & $239(35.7)$ & $<.001$ \\
\hline To celiac axis & $199(6.0)$ & $20(2.2)$ & $67(6.3)$ & $66(10.0)$ & $46(6.9)$ & $<.001$ \\
\hline To SMA & $183(5.5)$ & $2(0.2)$ & $62(5.8)$ & $65(9.8)$ & $54(8.1)$ & $<.001$ \\
\hline To right renal artery & $299(9.0)$ & 0 & $138(12.9)$ & $87(13.2)$ & $74(11.1)$ & $<.001$ \\
\hline To left renal artery & $686(20.7)$ & $19(2.1)$ & $271(25.4)$ & $184(27.9)$ & $212(31.7)$ & $<.001$ \\
\hline With 4-branch prefabricated graft & $166(5.0)$ & $1(0.1)$ & $63(5.9)$ & $60(9.1)$ & $42(6.3)$ & $<.001$ \\
\hline Endarterectomy & $857(25.9)$ & $51(5.6)$ & $275(25.8)$ & $250(37.9)$ & $281(42.0)$ & $<.001$ \\
\hline Stenting & $264(8.0)$ & $15(1.6)$ & $86(8.1)$ & $73(11.1)$ & $90(13.5)$ & $<.001$ \\
\hline Endarterectomy or stenting & $940(28.4)$ & $58(6.3)$ & $303(28.4)$ & $270(40.9)$ & $309(46.2)$ & $<.001$ \\
\hline Endarterectomy, stenting, or bypass & $1360(41.1)$ & $88(9.6)$ & $510(47.8)$ & $357(54.1)$ & $405(60.5)$ & $<.001$ \\
\hline \multicolumn{7}{|l|}{ Adjuncts } \\
\hline Cerebrospinal fluid drainage $\dagger$ & $1484(44.8)$ & $471(51.5)$ & $647(60.7)$ & $260(39.4)$ & $106(15.8)$ & $<.001$ \\
\hline Left heart bypass & $1480(44.7)$ & $557(60.9)$ & $874(82.0)$ & $43(6.5)$ & $6(0.9)$ & $<.001$ \\
\hline LHB time, $\min$ & $\begin{array}{c}25 \pm 9 \\
(\mathrm{n}=1462)\end{array}$ & $\begin{array}{c}26 \pm 9 \\
(\mathrm{n}=553)\end{array}$ & $\begin{array}{c}24 \pm 9 \\
(\mathrm{n}=863)\end{array}$ & $\begin{array}{l}22 \pm 10 \\
(\mathrm{n}=40)\end{array}$ & $\begin{array}{r}27 \pm 23 \\
(\mathrm{n}=6)\end{array}$ & .001 \\
\hline $\mathrm{HCA}$ & $48(1.5)$ & $28(3.1)$ & $18(1.7)$ & $2(0.3)$ & 0 & $<.001$ \\
\hline Cold renal perfusion & $1934(58.4)$ & $158(17.3)$ & $701(65.8)$ & $511(77.4)$ & $564(84.3)$ & $<.001$ \\
\hline Selective visceral perfusion & $776(23.5)$ & $60(6.6)$ & $675(63.3)$ & $36(5.5)$ & $5(0.7)$ & $<.001$ \\
\hline
\end{tabular}

Values are $\mathrm{n}(\%)$, mean \pm standard deviation, or median [interquartile range]. TEVAR, Thoracic endovascular aortic repair; $E V A R$, endovascular aortic repair; $L S C A$, left subclavian artery; ICA/LA, intercostal/lumbar artery; SMA, superior mesenteric artery; $L H B$, left heart bypass; $H C A$, hypothermic circulatory arrest. *Full or partial extraction of endograft. Four extraction cases overlap by type of extraction, with simultaneous extraction of prior TEVAR and prior EVAR. †Intraoperative insertion of cerebrospinal fluid drainage catheter. Does not include postoperative use of cerebrospinal fluid drainage as a rescue measure. 
Tex). Continuous variables are presented as mean \pm standard deviation or median [interquartile range], as appropriate. Categorical variables are presented as number and percentage. Univariate comparisons were made with the Pearson chi-square test, Fisher exact test, or Kruskal-Wallis equality-of-populations rank test, as appropriate. All clinically relevant preoperative or intraoperative factors whose univariate association with outcomes had a $P$ value of $<.05$ were entered into multivariable logistic regression models to identify independent predictors; postoperative complications were not entered into models.

Variables selected for multivariable modeling of operative death and adverse event were age, degenerative aneurysm without dissection, chronic aortic dissection, coronary artery disease, cerebrovascular disease, chronic renal insufficiency, pulmonary disease, rupture, urgent or emergency repair, aortic clamp time, visceral artery procedure, HCA, connective tissue disorder, and peripheral vascular disease; additionally, the variables symptomatic and extent I, II, or IV TAAA repair were entered into the operative death model, and the variables acute symptoms and extent II TAAA repair were entered into the adverse event model. Variables selected for multivariable modeling of permanent renal failure were age, genetically triggered disorder, chronic aortic dissection, cerebrovascular disease, chronic renal insufficiency, serum creatinine, chronic obstructive pulmonary disease, acute symptoms, peripheral vascular disease, rupture, emergency repair, extent I repair, extent II repair, aortic clamping proximal to the left subclavian artery, aortic cross-clamp time, maximum unprotected ischemic time of left or right renal artery, visceral artery bypass, visceral artery procedure, iliac or femoral bypass, HCA, cold renal perfusion, and selective visceral perfusion. Variables selected for multivariate modeling of permanent paraplegia and permanent paraplegia or paraparesis after extent II TAAA repair were age, genetically triggered disorder, chronic aortic dissection, coronary artery disease, serum creatinine, past or current tobacco use, chronic symptoms, intercostal or lumbar artery reattachment, and visceral artery endarterectomy or stent. Variables selected for multivariate modeling of permanent paraplegia and permanent paraplegia or paraparesis after extent III TAAA repair were cerebrovascular disease, serum creatinine, past or current tobacco use, rupture, emergency repair, aortic cross-clamp time, visceral artery procedure, and selective visceral perfusion.

Stepwise models with a removal criterion of $P \geq .1$ were estimated, after which multinomial logistic regression models were generated to present parameter estimates as relative risk ratios (RRRs). Model fit was assessed with likelihood ratio chi-square tests, goodness-of-fit tests, and pseudo $\mathrm{R}^{2}$. During modeling, 272 missing values $(0.8 \%)$ for preoperative serum creatinine level were replaced with mean values ${ }^{23,24}$ based on matched categories of age, gender, and extent of repair, or with the postoperative creatinine value (if it was lower than the mean preoperative value). Survival over time and freedom from late events were estimated by the Kaplan-Meier or Cox regression methods.

\section{RESULTS \\ Early Outcomes}

There were 249 operative deaths $(7.5 \%$; Table 3$)$, which included 37 of 193 repairs performed in octogenarians (19.2\% mortality), 37 of 170 repairs involving rupture $(21.8 \%), 14$ of 439 repairs in patients aged 50 years or less $(3.2 \%), 9$ of 288 repairs in patients with Marfan syndrome $(3.1 \%)$, and 58 of 1020 repairs of chronic dissection $(5.7 \%)$. In 2586 elective repairs, operative death occurred after 161 repairs $(6.2 \%$, Table 4$)$; in 723 urgent or emergency repairs, operative death occurred after 88 repairs $(12.2 \% ; P<.001)$. The rate of operative death differed among the 4 groups $(P=.002)$, being higher in extents II and III $(9.5 \%$ and $8.8 \%)$ than in extents I and IV $(5.9 \%$ and $5.4 \%$, respectively) (Table 3 ). The most common cause of operative death (Table 5) was MOF, which resulted in 173 deaths $(67.5 \%)$ and was classified as primary MOF in 55 cases $(31.8 \%)$ and as secondary MOF due to inciting complications in 118 cases $(68.2 \%)$. In addition, there were 5 early repair failures ( 3 fistulas and 2 graft infections) that resulted in operative death.

Permanent paraplegia and paraparesis occurred after 97 $(2.9 \%)$ and $81(2.4 \%)$ repairs, respectively; only 5 of 439 repairs $(1.1 \%)$ in patients aged $\leq 50$ years resulted in permanent paraplegia. Of 189 patients $(5.7 \%)$ with permanent renal failure, the majority $(\mathrm{n}=107 ; 56.6 \%)$ did not survive to hospital discharge. Permanent stroke was relatively uncommon $(n=74 ; 2.2 \%)$. Unsurprisingly, adverse event rates were highest after extent II repair $(\mathrm{n}=203 ; 19.0 \%)$ and lowest after extent IV repair ( $\mathrm{n}=67 ; 10.0 \% ; P<.001$ across all extents). Of the 3060 survivors, $229(6.9 \%)$ were discharged with a lifealtering complication; of these, renal failure was the most common $(\mathrm{n}=82[2.5 \%])$, followed by paraparesis $(\mathrm{n}=73[2.2 \%])$, paraplegia $(\mathrm{n}=52[1.6 \%])$, and stroke $(\mathrm{n}=35[1.1 \%])$.

Within our cohort, we found that the subset of patients with rupture $(\mathrm{n}=170)$ included significantly more octogenarians $(n=25[14.7 \%])$ than the subset of patients without rupture $(\mathrm{n}=168[5.4 \%] ; P<.001)$, and the patients with rupture more often had prior repair failure $(\mathrm{n}=14[8.2 \%]$ vs $\mathrm{n}=122[3.9 \%], P=.015)$. In octogenarians $(\mathrm{n}=193)$, operative death $(\mathrm{n}=37$ $[19.2 \%]$ ) varied significantly by extent of repair $(P<.001)$, which our prior publication also noted ${ }^{25}$; the rate of operative death was greatest in extent II repair $(\mathrm{n}=14 / 38[36.8 \%])$ and lowest in extent IV repair $(n=6 / 61[9.8 \%])$. In octogenarians, the need for repair is often unexpected-more than one third of octogenarians underwent urgent or emergency repair $(n=69$ $[35.8 \%])$, compared with only one fifth of nonoctogenarians $(\mathrm{n}=654[21.0 \%], P<.001)$.

In regard to the need for urgent or emergency repair, median aortic diameter was approximately $1 \mathrm{~cm}$ larger in patients who required such repair $(6.9[5.9-8.0] \mathrm{cm})$ than in those who underwent elective repair $(6.1[5.5-7.0] \mathrm{cm}$; $P<.001)$. We compared preoperative and other characteristics between patients undergoing repair for aortic dissection $(\mathrm{n}=1185)$ versus degenerative aneurysm without dissection $(\mathrm{n}=2124)$ (Table 6); patients in the dissection cohort were younger, more often had connective tissue disorders, and, in general, were healthier. Of note, the median diameter at the time of repair was similar between groups $(6.2$ [5.5-7.0] $\mathrm{cm}$ vs 6.3 [5.6-7.2] $\mathrm{cm})$, but extent II repair was significantly more frequent in patients with dissection $(44.6 \%)$ than in patients with aneurysm without dissection $(25.3 \%)$. 
TABLE 3. Early outcomes

\begin{tabular}{|c|c|c|c|c|c|c|}
\hline Variable & $\begin{array}{c}\text { All } \\
\mathbf{n}=\mathbf{3 3 0 9}\end{array}$ & $\begin{array}{l}\text { Extent I } \\
\mathrm{n}=914\end{array}$ & $\begin{array}{l}\text { Extent II } \\
n=1066\end{array}$ & $\begin{array}{c}\text { Extent III } \\
n=660\end{array}$ & $\begin{array}{c}\text { Extent IV } \\
n=669\end{array}$ & $P$ value \\
\hline Adverse event* & $478(14.4)$ & $98(10.7)$ & $203(19.0)$ & $110(16.7)$ & $67(10.0)$ & $<.001$ \\
\hline Operative death & $249(7.5)$ & $54(5.9)$ & $101(9.5)$ & $58(8.8)$ & $36(5.4)$ & .002 \\
\hline In-hospital & $237(7.2)$ & $50(5.5)$ & $97(9.1)$ & $56(8.5)$ & $34(5.1)$ & .001 \\
\hline \multirow[t]{2}{*}{$30-\mathrm{d} \dagger$} & $159(4.9)$ & $34(3.8)$ & $68(6.5)$ & $34(5.2)$ & $23(3.4)$ & .01 \\
\hline & $(\mathrm{n}=3275)$ & $(\mathrm{n}=899)$ & $(\mathrm{n}=1052)$ & $(\mathrm{n}=656)$ & $(\mathrm{n}=668)$ & \\
\hline During operation & $11(0.3)$ & $6(0.7)$ & $3(0.3)$ & $2(0.3)$ & 0 & .2 \\
\hline Cerebral complication (composite) & $267(8.1)$ & $74(8.1)$ & $124(11.6)$ & $40(6.1)$ & $29(4.3)$ & $<.001$ \\
\hline Encephalopathy & $147(4.4)$ & $37(4.0)$ & $68(6.4)$ & $27(4.1)$ & $15(2.2)$ & .001 \\
\hline Intracranial hemorrhage & $29(0.9)$ & $6(0.7)$ & $15(1.4)$ & $6(0.9)$ & $2(0.3)$ & .09 \\
\hline Seizure & $32(1.0)$ & $7(0.8)$ & $15(1.4)$ & $8(1.2)$ & $2(0.3)$ & .1 \\
\hline Stroke & $98(3.0)$ & $33(3.6)$ & $46(4.3)$ & $9(1.4)$ & $10(1.5)$ & $<.001$ \\
\hline Permanent $\ddagger$ & $74(2.2)$ & $22(2.4)$ & $36(3.4)$ & $7(1.1)$ & $9(1.3)$ & .004 \\
\hline Temporary & $24(0.7)$ & $11(1.2)$ & $10(0.9)$ & $2(0.3)$ & $1(0.1)$ & .04 \\
\hline Hemorrhagic & $19(0.6)$ & $6(0.7)$ & $11(1.0)$ & 0 & $2(0.3)$ & .03 \\
\hline Ischemic or embolic & $82(2.5)$ & $27(3.0)$ & $38(3.6)$ & $9(1.4)$ & $8(1.2)$ & .003 \\
\hline Transient ischemic attack & $8(0.2)$ & $2(0.2)$ & $3(0.3)$ & $1(0.2)$ & $2(0.3)$ & .9 \\
\hline Spinal cord deficit (composite) & $317(9.6)$ & $71(7.8)$ & $147(13.9)$ & $71(10.8)$ & $27(4.0)$ & $<.001$ \\
\hline Permanent $\ddagger$ SCD & $178(5.4)$ & $31(3.4)$ & $85(8.0)$ & $46(7.0)$ & $16(2.4)$ & $<.001$ \\
\hline Permanent $\dagger$ paraplegia & $97(2.9)$ & $12(1.3)$ & $50(4.7)$ & $29(4.4)$ & $6(0.9)$ & $<.001$ \\
\hline Immediate & $50(1.5)$ & $6(0.7)$ & $26(2.4)$ & $14(2.1)$ & $4(0.6)$ & .001 \\
\hline Delayed & $47(1.4)$ & $6(0.7)$ & $24(2.3)$ & $15(2.3)$ & $2(0.3)$ & .001 \\
\hline Permanent $\ddagger$ paraparesis & $81(2.4)$ & $19(2.1)$ & $35(3.3)$ & $17(2.6)$ & $10(1.5)$ & .1 \\
\hline Immediate & $29(0.9)$ & $3(0.3)$ & $18(1.7)$ & $4(0.6)$ & $4(0.6)$ & .006 \\
\hline Delayed & $52(1.6)$ & $16(1.8)$ & $17(1.6)$ & $13(2.0)$ & $6(0.9)$ & .006 \\
\hline Temporary SCD & $146(4.4)$ & $41(4.5)$ & $65(6.1)$ & $28(4.2)$ & $12(1.8)$ & $<.001$ \\
\hline Temporary paraparesis & $94(2.8)$ & $26(2.8)$ & $46(4.3)$ & $15(2.3)$ & $7(1.0)$ & .001 \\
\hline Temporary paraplegia & $52(1.6)$ & $15(1.6)$ & $19(1.8)$ & $13(2.0)$ & $5(0.7)$ & .3 \\
\hline Neurogenic bladder & $22(0.7)$ & $2(0.2)$ & $12(1.1)$ & $6(0.9)$ & $2(0.3)$ & .04 \\
\hline Acute renal dysfunction & $406(12.3)$ & $73(8.0)$ & $170(15.9)$ & $81(12.3)$ & $82(12.3)$ & $<.001$ \\
\hline Renal failure necessitating dialysis & $250(7.6)$ & $45(4.9)$ & $102(9.6)$ & $52(7.9)$ & $51(7.6)$ & .002 \\
\hline Permanent $\ddagger$ & $189(5.7)$ & $29(3.2)$ & $78(7.3)$ & $43(6.5)$ & $39(5.8)$ & .001 \\
\hline Temporary & $61(1.8)$ & $16(1.8)$ & $24(2.3)$ & $9(1.4)$ & $12(1.8)$ & .6 \\
\hline Cardiac complication (composite) & $860(26.0)$ & $221(24.2)$ & $334(31.3)$ & $158(23.9)$ & $147(22.0)$ & $<.001$ \\
\hline Atrial arrhythmia & $541(16.3)$ & $147(16.1)$ & $215(20.2)$ & $93(14.1)$ & $86(12.9)$ & $<.001$ \\
\hline Myocardial infarction & $41(1.2)$ & $12(1.3)$ & $13(1.2)$ & $10(1.5)$ & $6(0.9)$ & .8 \\
\hline Cardiac tamponade & $10(0.3)$ & $5(0.5)$ & $5(0.5)$ & 0 & 0 & .08 \\
\hline Drainage of pericardial effusion & $20(0.6)$ & $7(0.8)$ & $9(0.8)$ & $1(0.2)$ & $3(0.4)$ & .3 \\
\hline Pulmonary complication (composite) & $1185(35.8)$ & $347(38.0)$ & $455(42.7)$ & $223(33.8)$ & $160(23.9)$ & $<.001$ \\
\hline Respiratory failure & $830(25.1)$ & $225(24.6)$ & $330(31.0)$ & $159(24.1)$ & $116(17.3)$ & $<.001$ \\
\hline Necessitating tracheostomy & $281(8.5)$ & $74(8.1)$ & $131(12.3)$ & $46(7.0)$ & $30(4.5)$ & $<.001$ \\
\hline ARDS & $140(4.2)$ & $41(4.5)$ & $63(5.9)$ & $20(3.0)$ & $16(2.4)$ & .001 \\
\hline Atelectasis necessitating bronchoscopy & $333(10.1)$ & 109 (11.9) & $135(12.7)$ & $52(7.9)$ & $37(5.5)$ & $<.001$ \\
\hline Chylothorax & $33(1.0)$ & $11(1.2)$ & $8(0.8)$ & $8(1.2)$ & $6(0.9)$ & .7 \\
\hline Drainage of pleural effusion & $233(7.0)$ & $81(8.9)$ & $83(7.8)$ & $49(7.4)$ & $20(3.0)$ & $<.001$ \\
\hline Pneumonia & $225(6.8)$ & $62(6.8)$ & $88(8.3)$ & $41(6.2)$ & $34(5.1)$ & .07 \\
\hline Evacuation of pneumothorax & $68(2.1)$ & $19(2.1)$ & $25(2.3)$ & $15(2.3)$ & $9(1.3)$ & .5 \\
\hline Pulmonary embolism & $28(0.8)$ & $9(1.0)$ & $8(0.8)$ & $5(0.8)$ & $6(0.9)$ & .9 \\
\hline Reintubation & 479 (14.5) & $130(14.2)$ & $190(17.8)$ & $99(15.0)$ & $60(9.0)$ & $<.001$ \\
\hline Left vocal cord paralysis & $502(15.2)$ & $239(26.1)$ & $248(23.3)$ & $9(1.4)$ & $6(0.9)$ & $<.001$ \\
\hline Bleeding requiring reoperation & $110(3.3)$ & $33(3.6)$ & $40(3.8)$ & $20(3.0)$ & $17(2.5)$ & .5 \\
\hline Gastrointestinal complication (composite) & $126(3.8)$ & $20(2.2)$ & $45(4.2)$ & $30(4.5)$ & $31(4.6)$ & .03 \\
\hline Gastrointestinal ischemia & $31(0.9)$ & $2(0.2)$ & $11(1.0)$ & $9(1.4)$ & $9(1.3)$ & .05 \\
\hline Pancreatitis & $9(0.3)$ & $1(0.1)$ & $4(0.4)$ & $2(0.3)$ & $2(0.3)$ & .7 \\
\hline Gastrointestinal bleeding & $63(1.9)$ & $8(0.9)$ & $23(2.2)$ & $19(2.9)$ & $13(1.9)$ & .03 \\
\hline Gastrointestinal obstruction & $37(1.1)$ & $10(1.1)$ & $10(0.9)$ & $4(0.6)$ & $13(1.9)$ & .1 \\
\hline
\end{tabular}


TABLE 3. Continued

\begin{tabular}{|c|c|c|c|c|c|c|}
\hline Variable & $\begin{array}{c}\text { All } \\
\mathbf{n}=\mathbf{3 3 0 9}\end{array}$ & $\begin{array}{l}\text { Extent I } \\
n=914\end{array}$ & $\begin{array}{l}\text { Extent II } \\
\mathrm{n}=1066\end{array}$ & $\begin{array}{c}\text { Extent III } \\
\mathbf{n}=\mathbf{6 6 0}\end{array}$ & $\begin{array}{c}\text { Extent IV } \\
\mathbf{n}=\mathbf{6 6 9}\end{array}$ & $P$ value \\
\hline Sepsis & $148(4.5)$ & $34(3.7)$ & $60(5.6)$ & $29(4.4)$ & $25(3.7)$ & .1 \\
\hline Wound infection & $108(3.3)$ & $31(3.4)$ & $51(4.8)$ & $17(2.6)$ & $9(1.3)$ & .004 \\
\hline Discharge to long-term acute care & $274(8.3)$ & $61(6.7)$ & $113(10.6)$ & $60(9.1)$ & $40(6.0)$ & .001 \\
\hline Early survival with life-altering complication $\S$ & $229(6.9)$ & $44(4.8)$ & $102(9.6)$ & $52(7.9)$ & $31(4.6)$ & $<.001$ \\
\hline Survival with renal failure (dialysis) & $82(2.5)$ & $12(1.3)$ & $36(3.4)$ & $17(2.6)$ & $17(2.5)$ & .03 \\
\hline Survival with permanent stroke & $35(1.1)$ & $9(1.0)$ & $18(1.7)$ & $4(0.6)$ & $4(0.6)$ & .08 \\
\hline Survival with permanent paraplegia & $52(1.6)$ & $7(0.8)$ & $25(2.3)$ & $17(2.6)$ & $3(0.4)$ & .001 \\
\hline Survival with permanent paraparesis & $73(2.2)$ & $17(1.9)$ & $31(2.9)$ & $16(2.4)$ & $9(1.3)$ & .1 \\
\hline Early survivors $\|:$ length of intensive care unit stay, $\mathrm{d}$ & $4[3-6]$ & $4[3-7]$ & $4[3-8]$ & $4[3-6]$ & $3[2-5]$ & $<.001$ \\
\hline Early survivors $\|$ : length of hospital stay, d & $12[9-17]$ & $12[9-17]$ & $13[10-20]$ & $11[9-15]$ & $10[8-13]$ & $<.001$ \\
\hline
\end{tabular}

Values are $\mathrm{n}(\%)$ or median [interquartile range]. ARDS, Acute respiratory distress syndrome. *Adverse event is defined as operative death or permanent (present at hospital

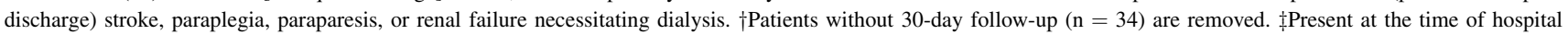
discharge or early death. $\S$ Discharged with stroke, paraplegia, paraparesis, or renal failure necessitating dialysis $(\mathrm{n}=3060)$. $\|$ Early survivors $(\mathrm{n}=3060)$

Independent predictors of adverse event, permanent renal failure, permanent paraplegia or paraparesis, and operative death are shown in Tables 7, 8, and 9. Regarding adverse event, permanent renal failure, and operative death, greater age (by each year), rupture, the need for a visceral artery procedure, and HCA were associated with greater risk. In the operative death model, extent IV repair was associated with a $50 \%$ reduction in risk $(\mathrm{RRR}=0.5, P=.001)$. Extent II repair was associated with increased risk in the adverse event and permanent renal failure models $(\mathrm{RRR}=1.49$, $P=.001 ; \mathrm{RRR}=1.39, P=.05$, respectively). In regard to modeling for paraplegia, we developed separate analyses for extents II and III, which were associated with higher rates of paraplegia than extents I and IV (4.7\% and $4.4 \%$ vs $1.3 \%$ and $0.9 \%$, respectively); notably, independent predictors (Table 8) were different between the 2 paraplegia models. For patients who underwent extent II repairs, coronary artery disease and chronic symptoms increased the risk of paraplegia; however, having a genetically triggered disorder or the intraoperative reattachment of intercostal or lumbar arteries decreased risk. For patients who underwent extent III repairs, cerebrovascular disease, emergency repair, and selective perfusion of the visceral arteries were associated with increased risk of paraplegia—no characteristics were associated with decreased risk.

To assess the effects of era on surgical outcomes, we stratified the data and applied the overall operative mortality multivariable model to all repairs by surgical era (Table 9); only age at repair was significantly predictive of operative mortality in all 3 eras. In Era 1, chronic renal insufficiency was highly predictive of operative death, but it was less predictive in Eras 2 and 3. In Era 2, rupture was highly predictive of operative death, but it was less so in Era 3. In Era 3, preoperative pulmonary disease played a considerably larger role than it did in Eras 1 and 2. We then used the overall operative mortality model to determine the predicted probability of operative death for Eras 1, 2, and 3. The predicted operative mortality rates by era were $5.4 \%$ (Era 1), 4.9\% (Era 2), and 6.4\% (Era 3). Unfortunately, the observed/expected frequency ratios were greater than 1 for each era (Era $1=1.3$; Era $2=1.4$; Era $3=1.4$ ), indicating that the model underpredicted operative mortality.

TABLE 4. Results of consecutive elective cases $(n=2586)$

\begin{tabular}{|c|c|c|c|c|c|c|}
\hline Variable & $\begin{array}{c}\text { All } \\
\mathbf{n}=\mathbf{2 5 8 6}\end{array}$ & $\begin{array}{l}\text { Extent I } \\
n=700\end{array}$ & $\begin{array}{c}\text { Extent II } \\
\mathbf{n}=866\end{array}$ & $\begin{array}{c}\text { Extent III } \\
\mathbf{n}=\mathbf{5 0 4}\end{array}$ & $\begin{array}{c}\text { Extent IV } \\
n=516\end{array}$ & $P$ value \\
\hline Adverse event & $329(12.7)$ & $63(9.0)$ & $154(17.8)$ & $73(14.5)$ & $39(7.6)$ & $<.001$ \\
\hline Operative mortality & $161(6.2)$ & $32(4.6)$ & $72(8.3)$ & $41(8.1)$ & $16(3.1)$ & $<.001$ \\
\hline Permanent paraplegia* & $66(2.6)$ & $8(1.1)$ & $37(4.3)$ & $18(3.6)$ & $3(0.6)$ & $<.001$ \\
\hline Permanent paraparesis* & $57(2.2)$ & $14(2.0)$ & $25(2.9)$ & $10(2.0)$ & $8(1.6)$ & .4 \\
\hline Permanent renal failure necessitating dialysis* & $132(5.1)$ & $17(2.4)$ & $64(7.4)$ & $28(5.6)$ & $23(4.5)$ & $<.001$ \\
\hline Permanent stroke* & $60(2.3)$ & $17(2.4)$ & $31(3.6)$ & $5(1.0)$ & $7(1.4)$ & .007 \\
\hline Survival with life-altering complication $\dagger$ & $168(6.5)$ & $31(4.4)$ & $82(9.5)$ & $32(6.3)$ & $23(4.5)$ & $<.001$ \\
\hline
\end{tabular}

Values are $\mathrm{n}(\%)$. Outcomes of interest (paraplegia, paraparesis, renal failure necessitating dialysis, and stroke) are permanent complications present at discharge or present in those patients with early death. *Excludes 5 patients who died during the operation. $\dagger$ Discharge with permanent paraplegia, paraparesis, renal failure, or stroke in 2425 early survivors of elective repair. 
TABLE 5. Causes of early death $(n=249$ of 3309 patients $)$

\begin{tabular}{lc}
\hline \multicolumn{1}{c}{ Cause } & No. $(\%)$ \\
\hline Multisystem organ failure & $173(67.5)$ \\
$\quad$ Primary $(\mathrm{n}=55)$ & \\
$\quad$ Secondary $(\mathrm{n}=118)$ & $28(11.2)$ \\
Cardiac failure & $13(5.2)$ \\
Respiratory failure & $10(4.0)$ \\
Stroke & $9(3.6)$ \\
Unknown & $5(2.0)$ \\
Exsanguination & $4(1.6)$ \\
Gastrointestinal bleed & $4(1.6)$ \\
Pulmonary embolism & $1(0.4)$ \\
Neoplasm & $1(0.4)$ \\
Intracranial hemorrhage & $1(0.4)$ \\
Suicide &
\end{tabular}

\section{Late Outcomes}

There were 1864 late deaths. Estimated survival was $83.5 \% \pm 0.7 \%$ at 1 year, $63.6 \% \pm 0.9 \%$ at 5 years, $36.8 \% \pm 1.0 \%$ at 10 years, and $18.3 \% \pm 0.9 \%$ at 15 years (Figure 3, $A$ ). Over time, there were 88 late repair failures; these included failures with overlapping causes, such as graft infection $(\mathrm{n}=18)$, pseudoaneurysm formation $(\mathrm{n}=43)$, and patch aneurysm $(\mathrm{n}=33)$. Freedom from repair failure was $97.9 \% \pm 0.3 \%$ at 5 years, $95.3 \% \pm 0.6 \%$ at 10 years, and $94.1 \% \pm 0.8 \%$ at 15 years (Figure 3, B). Of these 88 patients, 44 had aortic reintervention; 29 of these 88 patients $(33.0 \%)$ remain alive. Patients with chronic dissection had significantly higher survival rates than patients without aortic dissection (Figure 3,C), even after correction for age (using the mean age, 64.6 years) (Figure 3,D).

\section{DISCUSSION}

Patients with TAAA face an extremely difficult clinical situation. ${ }^{26}$ They risk life-threatening rupture without TAAA repair; however, repair itself carries substantial risk, including risk of death or, in operative survivors, life-altering complications such as permanent stroke, paraplegia, and renal failure necessitating dialysis. We and others ${ }^{7,13,27-29}$ long ago established that the postoperative risks vary by the extent of TAAA repair. Rates of early mortality range from $5 \%$ to $8 \%$ for extent I, $8 \%$ to $13 \%$ for extent II, $8 \%$ to $21 \%$ for extent III, and $2 \%$ to $6 \%$ for extent IV at experienced centers $^{27,30-32}$; likewise, our results vary significantly by extent of repair $(\mathrm{I}=5.9 \%$, II $=9.5 \%$, III $=8.8 \%$, IV $=5.4 \% ; P=.002)$. As expected, we found that

TABLE 6. Comparison of preoperative and other factors in aortic dissection $(n=1185)$ versus aneurysm without dissection $(n=2124)$

\begin{tabular}{|c|c|c|c|c|}
\hline Variable & $\begin{array}{c}\text { All } \\
\mathbf{n}=\mathbf{3 3 0 9}\end{array}$ & $\begin{array}{l}\text { Aortic dissection } \\
\quad \mathbf{n}=\mathbf{1 1 8 5} \\
\end{array}$ & $\begin{array}{l}\text { Aneurysm without dissection } \\
\qquad \mathrm{n}=\mathbf{2 1 2 4} \\
\end{array}$ & $P$ value \\
\hline Age (y) & $67[59-73]$ & $58[48-68]$ & $70[65-75]$ & $<.001$ \\
\hline Age $\leq 50$ y & $439(13.3)$ & 354 (29.9) & $85(4.0)$ & $<.001$ \\
\hline Age $>79$ y & $193(5.8)$ & $24(2.0)$ & $169(8.0)$ & $<.001$ \\
\hline Maximum distal aortic diameter, $\mathrm{cm}$ & $\begin{array}{c}6.2[5.5-7.2] \\
(\mathrm{n}=2936)\end{array}$ & $\begin{array}{l}6.2[5.5-7.0] \\
(\mathrm{n}=1077)\end{array}$ & $\begin{array}{c}6.3[5.6-7.2] \\
(\mathrm{n}=1859)\end{array}$ & .1 \\
\hline Genetically triggered disorder & $523(15.8)$ & $427(36.0)$ & $96(4.5)$ & $<.001$ \\
\hline Connective tissue disorder & $330(10.0)$ & $303(25.6)$ & $27(1.3)$ & $<.001$ \\
\hline Hypertension & $2805(84.8)$ & 992 (83.7) & $1813(85.4)$ & .2 \\
\hline Hyperlipidemia & $967(29.2)$ & $261(22.0)$ & $706(33.2)$ & $<.001$ \\
\hline Diabetes & $261(7.9)$ & $72(6.1)$ & $189(8.9)$ & .004 \\
\hline Coronary artery disease & $1232(37.2)$ & $304(25.7)$ & $928(43.7)$ & $<.001$ \\
\hline Prior myocardial infarction & $652(19.7)$ & $156(13.2)$ & $496(23.4)$ & $<.001$ \\
\hline Pulmonary disease & $1298(39.2)$ & $293(24.7)$ & $1005(47.3)$ & $<.001$ \\
\hline Cerebrovascular disease & $575(17.4)$ & $163(13.8)$ & $412(19.4)$ & $<.001$ \\
\hline Prior stroke & $334(10.1)$ & $110(9.3)$ & $224(10.5)$ & .3 \\
\hline Peripheral vascular disease & $885(25.8)$ & $184(15.5)$ & 671 (31.6) & $<.001$ \\
\hline Past or current tobacco use & $2616(79.1)$ & $751(63.4)$ & $1865(87.8)$ & $<.001$ \\
\hline Chronic renal insufficiency & $99(3.0)$ & $28(2.4)$ & $71(3.3)$ & .1 \\
\hline Chronic renal failure (dialysis) & $60(1.8)$ & $18(1.5)$ & $42(2.0)$ & .4 \\
\hline Symptomatic & $2117(64.0)$ & $783(66.1)$ & $1334(62.8)$ & .06 \\
\hline Acute symptoms & $549(16.6)$ & $252(21.3)$ & $297(14.0)$ & $<.001$ \\
\hline Chronic symptoms & $1664(50.3)$ & $567(47.8)$ & 1097 (51.6) & .04 \\
\hline Rupture & $170(5.1)$ & $42(3.5)$ & $128(6.0)$ & .002 \\
\hline Urgent or emergency repair & $723(21.8)$ & $304(25.7)$ & 419 (19.7) & $<.001$ \\
\hline Extent II repair & $1066(32.3)$ & $528(44.6)$ & $538(25.3)$ & $<.001$ \\
\hline Redo thoracotomy & $563(17.0)$ & $190(16.0)$ & 373 (17.6) & .3 \\
\hline
\end{tabular}

Values are $\mathrm{n}(\%)$ or median [interquartile range]. 
TABLE 7. Relative risk ratios for preoperative and operative predictors of adverse events $(n=481)$ and permanent renal failure $(\mathbf{n}=190)$ among all patients

\begin{tabular}{|c|c|c|}
\hline \multirow[b]{2}{*}{ Variable } & \multicolumn{2}{|c|}{ Outcome } \\
\hline & RRR (CI) & $P$ value \\
\hline \multicolumn{3}{|l|}{ Adverse event model } \\
\hline $\mathrm{HCA}$ & $4.16(2.09-8.28)$ & $<.001$ \\
\hline Rupture & $2.42(1.61-3.62)$ & $<.001$ \\
\hline Chronic renal insufficiency & $2.32(1.45-3.71)$ & $<.001$ \\
\hline Extent II TAAA repair & $1.49(1.18-1.88)$ & .001 \\
\hline Urgent or emergency repair & $1.42(1.10-1.84)$ & .007 \\
\hline $\begin{array}{l}\text { Endarterectomy, stenting, or bypass } \\
\text { of visceral arteries }\end{array}$ & $1.36(1.11-1.68)$ & .004 \\
\hline Cerebrovascular disease & $1.24(0.97-1.60)$ & .09 \\
\hline Age, y & $1.05(1.03-1.06)$ & $<.001$ \\
\hline Aortic clamp time, min* & $1.02(1.01-1.02)$ & $<.001$ \\
\hline Connective tissue disorder & $0.60(0.34-1.05)$ & .07 \\
\hline \multicolumn{3}{|l|}{ Permanent renal failure model } \\
\hline $\mathrm{HCA}$ & $3.16(1.15-8.68)$ & .03 \\
\hline Rupture & $2.66(1.64-4.32)$ & $<.001$ \\
\hline $\begin{array}{l}\text { Endarterectomy, stent, or bypass } \\
\text { of visceral arteries }\end{array}$ & $1.53(1.08-2.17)$ & .02 \\
\hline Extent II TAAA repair & $1.39(0.99-1.95)$ & .054 \\
\hline $\begin{array}{l}\text { Preoperative serum creatinine } \\
\text { level, } \mathrm{mg} / \mathrm{dL} \dagger\end{array}$ & $1.38(1.22-1.55)$ & $<.001$ \\
\hline Age, y & $1.06(1.05-1.08)$ & $<.001$ \\
\hline $\begin{array}{l}\text { Maximum left or right renal artery } \\
\text { unprotected ischemic time, } \min ^{*}, \downarrow\end{array}$ & $1.02(1.01-1.03)$ & $<.001$ \\
\hline Aortic clamp time, min* & $1.01(1.00-1.01)$ & .007 \\
\hline
\end{tabular}

RRR, Relative risk ratio; $C I$, confidence interval; $H C A$, hypothermic circulatory arrest; TAAA, thoracoabdominal aortic aneurysm. *Variable is not thought to make a substantial clinical contribution, because the RRR approaches 1 . $†$ Missing preoperative serum creatinine values were substituted with mean values that were matched by age, gender, and extent of TAAA repair. However, if the peak postoperative value was lower than the matched mean value, then this value was used. $\ddagger$ The greater value of either renal artery. Unprotected ischemic time is the total ischemic time minus left heart bypass time. For continuous data, RRR is per unit.

extent II repairs confer the greatest overall risk of mortality and morbidity. ${ }^{33}$

However, it is clear that risk stems from more than just the amount of aorta replaced during repair and that patient-specific variables also play a substantial role in poor postoperative outcomes. Going further, the process by which extensive aneurysms (extents I and II) form often is different from what occurs in the development of less-extensive aneurysms (extents III and IV). Of note, we found that patients with dissection generally had fewer comorbidities than patients with degenerative aneurysm without dissection (Table 6), with the caveat that some of the health conditions we studied (eg, hyperlipidemia, coronary artery disease, cerebrovascular disease) are typically associated with the aging process itself. Clearly, there are select groups of patients who should expect good to excellent outcomes after even the most extensive TAAA repair (extent II repair); typically, this includes patients aged 50 years or less, patients with connective tissue disorders, ${ }^{34,35}$ and to a lesser extent, patients with chronic aortic dissection. ${ }^{36}$ Likewise, there are select groups of patients for whom extent II TAAA repair might exceed a reasonable level of risk, such as octogenarians. $^{25,37,38}$

Age remains a powerful predictor of poor postoperative outcomes, and greater age is an independent predictor of adverse event, operative mortality, and permanent renal failure. Likewise, rupture is an independent predictor of adverse events, operative mortality, and permanent renal failure; rupture predicts the patient's risk of permanent renal failure more strongly than it predicts operative mortality or adverse events, more than doubling the risk $(\mathrm{RRR}=2.66)$.

In regard to permanent spinal cord deficits, approximately half of all cases of permanent paraplegia occurred in a delayed fashion and developed after spinal cord function was initially found to be unimpaired; this observation did not differ among extents of repair. We observed that permanent paraplegia primarily affected patients who underwent extent II or III repair, but we noted that these 2 groups of patients had dissimilar preoperative characteristics. Subsequently, we developed separate paraplegia models for the extent II and III cohorts-partly because of this dissimilarity and partly because of speculation that in extent II repair, paraplegia results from the interruption and loss of spinal-perfusing arteries, whereas in extent III repair, embolism may play a larger role.

Although both paraplegia models underwent the same preliminary analysis, the final models did not overlap. Among other factors, we found reattachment of intercostal or lumbar arteries in extent II repair to be an independent predictor of substantially lower risk of permanent paraplegia $(\mathrm{RRR}=0.46, P=.03)$. In contrast, paraplegia after extent III repair was independently predicted by cerebrovascular disease $(\mathrm{RRR}=3.08, P=.006)$, emergency repair $(\mathrm{RRR}=3.44, P=.01)$, and the use of selective visceral perfusion $(\mathrm{RRR}=8.11, P<.001)$. Cerebrovascular disease could be indicative of generalized atherosclerotic disease, which tends to be more common in the slightly older extent III cohort. When we expanded our analysis to include both permanent paraplegia and paraparesis, the extent II model was largely unchanged, but in the extent III model, the need for a visceral artery procedure (eg, endarterectomy, stent, or bypass; $\mathrm{RRR}=2.51, P=.009)$ replaced cerebrovascular disease in the model-the need for such procedures can be interpreted as a marker for atherosclerotic disease, which tends to increase embolic debris. Tanaka and colleagues ${ }^{39}$ recently identified embolism as a major cause of spinal cord deficit after distal aortic repair. In a small study, Zangrillo and coauthors ${ }^{40}$ found postoperative paraplegia concentrated in patients with extent II and III repairs; the authors speculated that in patients with spinal cord 
TABLE 8. Relative risk ratio for preoperative and operative predictors of permanent paraplegia or permanent paraparesis after extent II $(n=1066)$ or extent III $(n=660)$ repair

\begin{tabular}{|c|c|c|}
\hline \multirow[b]{2}{*}{ Variable } & \multicolumn{2}{|l|}{ Outcome } \\
\hline & RRR (CI) & $P$ value \\
\hline \multicolumn{3}{|c|}{ Permanent paraplegia after extent II TAAA repair $(n=50)$} \\
\hline Chronic symptoms of aortic disease & $3.00(1.56-5.75)$ & .001 \\
\hline Past or current tobacco use & $2.75(0.83-9.15)$ & .10 \\
\hline Coronary artery disease & $1.92(1.07-3.43)$ & .03 \\
\hline $\begin{array}{l}\text { Reattachment of intercostal or } \\
\text { lumbar arteries }\end{array}$ & $0.46(0.23-0.93)$ & .03 \\
\hline Genetically triggered disorder & $0.23(0.05-0.98)$ & .047 \\
\hline \multicolumn{3}{|c|}{ Permanent paraplegia or paraparesis after extent II TAAA repair $(n=85$} \\
\hline Chronic symptoms of aortic disease & $1.83(1.15-2.91)$ & .01 \\
\hline Coronary artery disease & $1.78(1.13-2.81)$ & .01 \\
\hline $\begin{array}{l}\text { Reattachment of intercostal or } \\
\text { lumbar arteries }\end{array}$ & $0.55(0.31-0.99)$ & .045 \\
\hline Genetically triggered disorder & $0.37(0.17-0.82)$ & .02 \\
\hline \multicolumn{3}{|c|}{ Permanent paraplegia after extent III TAAA repair $(n=29)$} \\
\hline Selective perfusion of visceral arteries & $8.11(3.06-21.45)$ & $<.001$ \\
\hline Emergent repair & $3.44(1.28-9.22)$ & .01 \\
\hline Cerebrovascular disease & $3.08(1.39-6.82)$ & .006 \\
\hline \multicolumn{3}{|c|}{ Permanent paraplegia or paraparesis after extent III TAAA repair $(n=46)$} \\
\hline Selective perfusion of visceral arteries & $5.61(2.40-13.12)$ & $<.001$ \\
\hline $\begin{array}{l}\text { Endarterectomy, stent, or bypass of } \\
\text { visceral arteries }\end{array}$ & $2.51(1.26-4.98)$ & .009 \\
\hline Emergency repair & $2.23(0.97-5.12)$ & .06 \\
\hline
\end{tabular}

circulation already damaged by arterial plaque or mural thrombi, aortic clamping may exacerbate spinal ischemia, which was evidenced by elevated levels of intrathecal lactate. Of note, in both of our paraplegia models, age was not predictive of permanent paraplegia, and, somewhat surprisingly, neither was rupture.
Although cerebrovascular disease was present in approximately one fifth of all patients $(n=575,17.4 \%)$, permanent stroke was a relatively infrequent postoperative complication in our series (overall rate of $2.2 \%$; the rate was lowest after extent III repairs [1.1\%] and highest after extent II repairs [3.4\%]). An analysis of National Surgical Quality Improvement Program data ${ }^{41}$ regarding 450 open surgical repairs of nonruptured TAAA found a similarly low overall rate of stroke $(2.2 \%)$. In single-center reports of TAAA repair, the incidence of stroke ranges from $1.9 \%$ to $8.9 \% .^{27,42,43}$ Several centers favor the routine use of HCA in TAAA repair, but such use typically does not include extent IV repairs, and a few of these centers further restrict standard HCA use to extent II TAAA repair. In centers that routinely use HCA, the contemporary incidence of stroke ranges from $3.7 \%$ to $6.3 \%{ }^{44,45}$

Pulmonary complication, a broad category composed of several variables (shown in Table 3), remained the most common complication, ${ }^{10,11,27,37,46}$ occurring after $35.8 \%$ of all TAAA repairs and $42.7 \%$ of extent II repairs. Pulmonary complications were expected because of the single-lung ventilation that extensive TAAA repair necessitates. Similar to our previously published results, ${ }^{10,11}$ cardiac complications occurred after $26.0 \%$ of TAAA repairs; again, in extent II repairs, the rate was higher $(31.3 \%)$. Many of these complications were cases of atrial arrhythmia (which occurred after $20.2 \%$ of extent II repairs), which is a poorly understood complication of TAAA repair. Paralysis of the left vocal cord is another common complication ${ }^{10,11}$; although the rate was $15.4 \%$ overall, it was higher $(26.1 \%)$ after extent I repair. In addition to causing hoarseness, left vocal cord paralysis can compromise pulmonary function; recurrent laryngeal nerve injury can result from localized

TABLE 9. Relative risk ratio stratified by surgical era for preoperative and operative predictors of operative mortality $(\mathbf{n}=\mathbf{2 4 9})$ after 3309 thoracoabdominal aortic aneurysm repairs

\begin{tabular}{|c|c|c|c|c|c|c|c|c|}
\hline \multirow[b]{2}{*}{ Variable } & \multicolumn{2}{|c|}{$\begin{array}{c}\text { Operative death } \\
\text { (nonstratified model) }\end{array}$} & \multicolumn{2}{|c|}{$\begin{array}{c}\text { Era 1 } \\
(\leq 1998)\end{array}$} & \multicolumn{2}{|c|}{$\begin{array}{c}\text { Era 2 } \\
(1999-2004) \\
\end{array}$} & \multicolumn{2}{|c|}{$\begin{array}{c}\text { Era 3 } \\
(2005-2014) \\
\end{array}$} \\
\hline & RRR (CI) & $P$ value & RRR (CI) & $P$ value & RRR (CI) & $P$ value & RRR (CI) & $P$ value \\
\hline $\mathrm{HCA}$ & $2.69(1.12-6.46)$ & .03 & $5.56 \times 10^{-6 *}$ & .9 & $2.50(0.46-13.62)$ & .3 & $3.90(1.27-11.94)$ & .02 \\
\hline Rupture & $2.38(1.47-3.83)$ & $<.001$ & $1.67(0.71-3.94)$ & .2 & $3.69(1.48-9.18)$ & .005 & $2.26(1.02-4.98)$ & .04 \\
\hline Chronic renal insufficiency & $1.98(1.09-3.56)$ & .02 & $2.91(1.03-8.24)$ & .045 & $1.16(0.33-4.10)$ & .8 & $2.32(0.91-5.91)$ & .08 \\
\hline Symptomatic at time of repair & $1.62(1.16-2.25)$ & .004 & $1.94(1.08-3.50)$ & .03 & $1.49(0.86-2.58)$ & .2 & $1.36(0.73-2.51)$ & .3 \\
\hline $\begin{array}{l}\text { Endarterectomy, stent, or bypass } \\
\text { of visceral arteries }\end{array}$ & $1.46(1.10-1.94)$ & .009 & $1.55(0.94-2.57)$ & .09 & $1.18(0.71-1.95)$ & .5 & $1.55(0.93-2.58)$ & .1 \\
\hline Urgent or emergency repair & $1.35(0.96-1.91)$ & .09 & $1.49(0.82-2.69)$ & .2 & $0.83(0.42-1.63)$ & .6 & $1.90(1.27-3.41)$ & .02 \\
\hline Cerebrovascular disease & $1.34(0.97-1.85)$ & .08 & $1.34(0.73-2.43)$ & .9 & $1.27(0.71-2.27)$ & .8 & $1.42(0.83-2.40)$ & .2 \\
\hline Pulmonary disease & $1.29(0.98-1.69)$ & .07 & $0.92(0.57-1.49)$ & .7 & $1.23(0.74-2.01)$ & .4 & $1.96(1.23-3.12)$ & .005 \\
\hline Age, y & $1.05(1.04-1.07)$ & $<.001$ & $1.05(1.02-1.08)$ & $<.001$ & $1.04(1.02-1.07)$ & .001 & $1.07(1.04-1.09)$ & $<.001$ \\
\hline Aortic clamp time, $\min \dagger$ & $1.01(1.01-1.02)$ & $<.001$ & $1.02(1.01-1.03)$ & .001 & $1.01(1.00-1.01)$ & .06 & $1.02(1.01-1.03)$ & $<.001$ \\
\hline Extent IV TAAA repair & $0.52(0.35-0.76)$ & .001 & $0.55(0.27-1.10)$ & .09 & $0.70(0.35-1.40)$ & .3 & $0.37(0.19-0.69)$ & .002 \\
\hline
\end{tabular}

$R R R$, Relative risk ratio; $C I$, confidence interval; $H C A$, hypothermic circulatory arrest; TAAA, thoracoabdominal aortic aneurysm. *Value is too small to be meaningful in Era 1 . $\dagger$ Variable is not thought to make a substantial clinical contribution, because the RRR approaches 1 . For continuous data, RRR is per unit. 

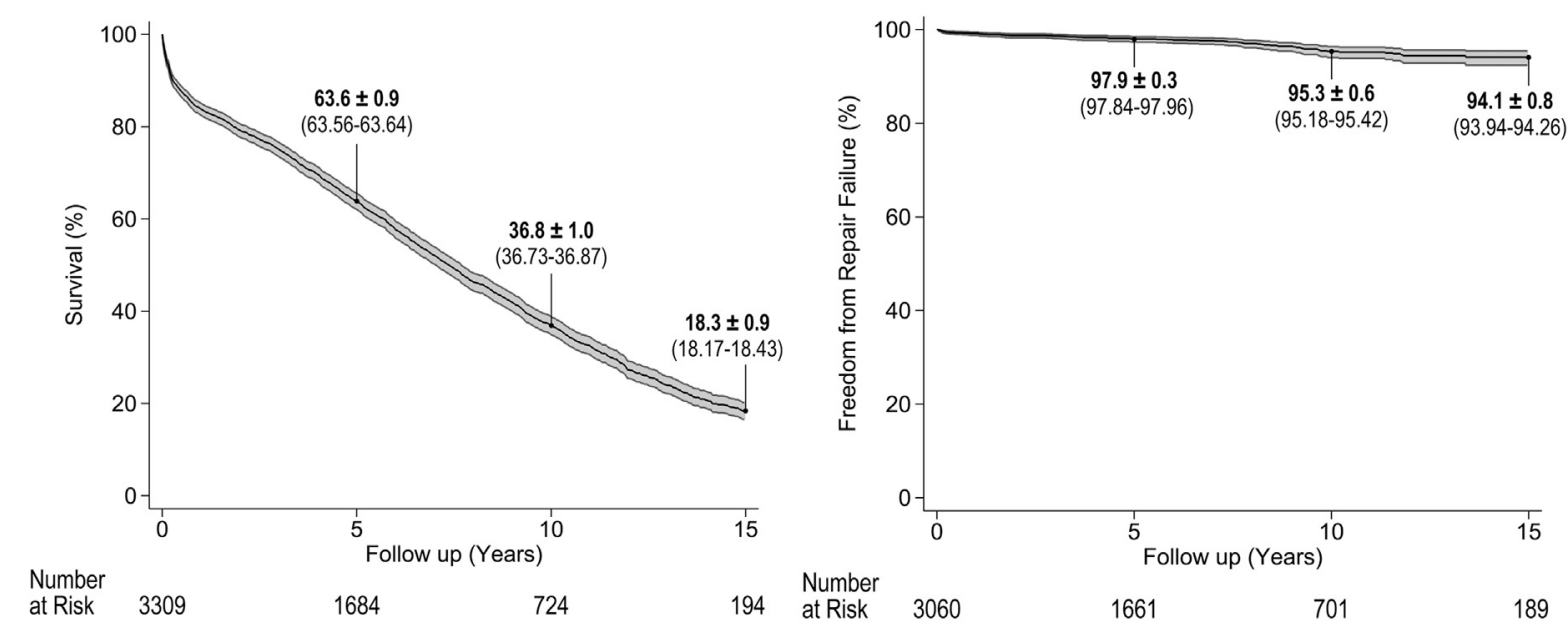

A

B
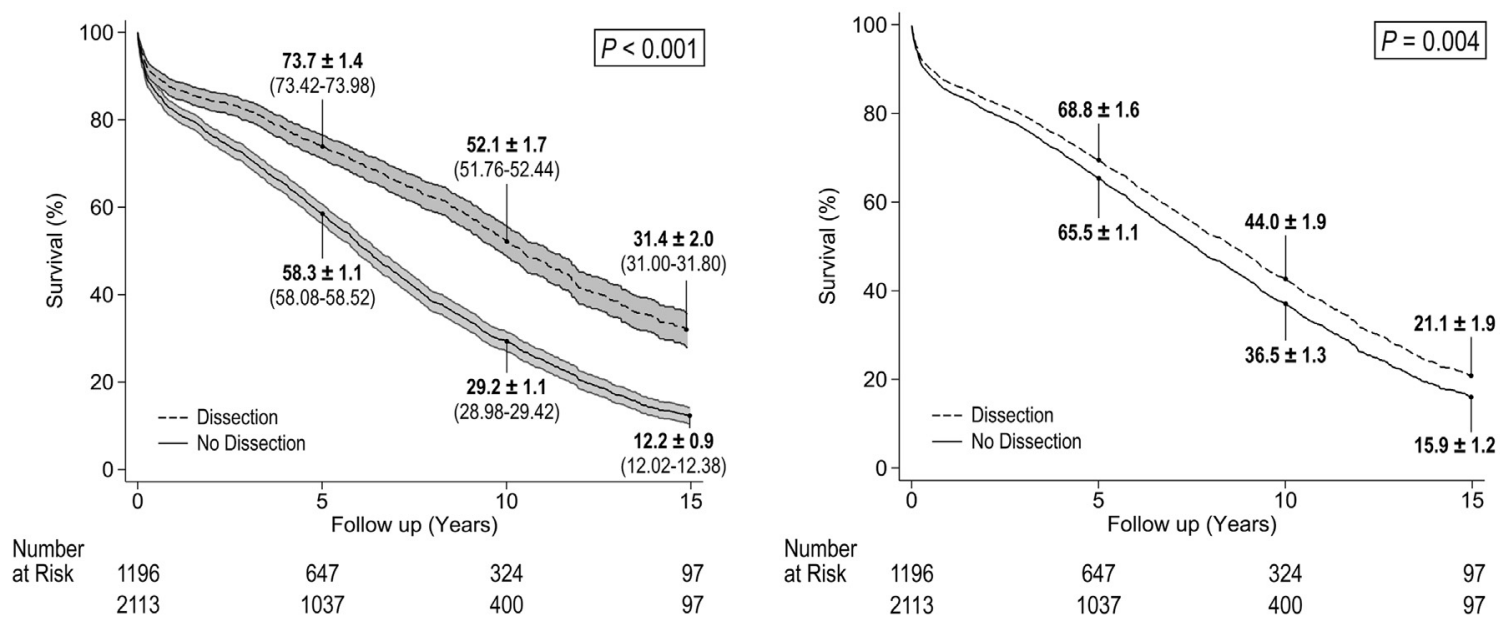

C

D

FIGURE 3. Kaplan-Meier curves of estimated survival (A) after 3309 TAAA repairs and (B) freedom from repair failure in 3060 early survivors. Survival was stratified by aortic dissection versus degenerative aneurysm without dissection (C). When we corrected for age (mean age, 64.6 years) by using a Cox regression model in analyzing survival after repair of aortic dissection versus degenerative aneurysm without dissection, (D) a survival advantage for patients with aortic dissection remained statistically significant $(P=.004)$. Confidence intervals $(95 \%)$ are shown in parentheses. TAAA, Thoracoabdominal aortic aneurysm.

trauma during exposure, aortic clamping, or suturing, especially when working proximal to the left subclavian artery in patients with chronic dissection.

Although our data show that open TAAA repair produces respectable outcomes when performed at an experienced center, there is clearly room for improvement. Surgical TAAA repair continues to pose substantial risks in the current era, particularly when the entire thoracoabdominal aorta (extent II) is replaced. It is certainly possible that incorporating additional interventions aimed at reducing rates of major complications would be of benefit to our practice, and we have actively explored doing so. For example, we briefly attempted to use motor-evoked potential monitoring techniques to reduce the incidence of spinal cord deficits, ${ }^{43}$ but because of logistical problems, we had difficulty obtaining consistently accurate, useful information, and we ultimately abandoned the effort. We will continue to explore the use of existing and investigational adjuncts in an effort to improve outcomes.

\section{Study Limitations}

Limitations of this study include its being a retrospective study from a single center. Although we collected prospective data during rounds for all patients who underwent TAAA repair since 2006, data for those who underwent operation before 2006 were collected solely by retrospective review of medical records. Thus, because of the inherent inadequacies of relying on past medical records, it is likely that before 2006 we did not fully capture preoperative characteristics or postoperative complications; this may have affected our predictive modeling efforts, including efforts to determine whether the surgical era 
$(1,2$, or 3$)$ at the time of repair affected adverse outcomes. Other authors have similarly reported the inability to detect sustained improvement in complication rates between surgical eras. ${ }^{46}$ Also, because of the large number of late deaths, we were unable to devote sufficient resources to determine causes for each death, so we may have undercaptured repair failure, and we were unable to determine the number of late deaths that resulted from aortic rupture. Although the use of HCA was found to be predictive of operative death, adverse event, and permanent renal failure, there is considerable selection bias regarding our use of $\mathrm{HCA}^{47}$ which we reserve for highly complex aortic repairs in which the proximal aorta is unclampable; thus, these findings are not generalizable to centers that use HCA as a standard technique. Likewise, in extent III repair, the selective use of visceral artery perfusion $(\mathrm{n}=36,5.5 \%)$ is biased toward atypically complex preoperative characteristics, so we suspect that its predictiveness of paraplegia or paraparesis in extent III repair is due to confounding factors and cannot be generalized to other aortic centers.

Although the dawn of endovascular TAAA (eTAAA) repair is clearly upon us, it is not yet clear whether this approach will benefit all patients or only select groups of them. In the United States, eTAAA remains an experimental approach, so its use here has been limited to a handful of centers. It is not clear what will happen when its use is broadened-real-world experience suggests that risk often increases when carefully crafted, industry-based criteria for use are loosened. At present, many eTAAA devices must be customized for the patient, which requires several weeks' notice, but off-the-shelf modular and fenestrated devices are in development. We know that compared with open TAAA repair, eTAAA repair is associated with lower operative mortality and length of hospitalization, whereas rates of mesenteric ischemia, paraplegia, branch-vessel occlusion, and late reintervention are often higher. Most pertinent, the reported rates of spinal cord deficit after extent II eTAAA have ranged as high as $30 \%$ to $50 \%{ }^{48,49}$

Comparison of open and eTAAA repair is complicated by the fact that Crawford extents of repair do not apply perfectly to eTAAA repairs. For example, an extent III TAAA open repair involves replacing the distal half of the descending thoracic aorta and a substantial portion of the abdominal aorta. In eTAAA for the same type of aneurysm, an even larger segment of the aorta needs to be covered (by approximately 4-6 cm, to secure proximal and distal landing zones), typically without revascularization of the intercostal and lumbar arteries; this can result in "upstaging" of the extent of repair, in that an aneurysm that would be treated by open extent III repair would need an extent II eTAAA repair because the area of coverage is extended across extent boundaries. Although efforts are being made to compensate for the resulting loss of perfusion by promoting spinal cord preconditioning or by creating intentional endoleaks ${ }^{50}$ this problem may prove to be a "real-world" limitation of eTAAA repair. In addition, one must consider that in certain subsets of younger patients, extent II TAAA repair can be performed at low risk of adverse outcomes. We know that in elderly patients, in whom extent III and IV TAAA repairs are common, the need for repair is often unexpected-more than one third of these patients' repairs are performed urgently or emergently, often in response to acute symptoms or rupture. These patients may benefit from off-the-shelf eTAAA devices, with the caveat that these patients commonly have severe atherosclerosis, and embolic debris may be discharged during repair, potentially causing stroke or paraplegia. Thus, the preferential adoption of eTAAA should be tempered by caution, and a balance should be sought with open repair. In the future, a larger challenge may be maintaining sufficient skill among aortic surgeons to perform open TAAA repair safely and effectively when it is needed.

\section{CONCLUSIONS}

We have presented our single-practice experience with TAAA repair, which spans approximately 3 decades. Although we have refined our operative technique over time in an effort to prevent early death and life-altering complications, continued improvement is needed. A better understanding of preoperative risk factors may enhance efforts to optimize patient health before repair, but the low-hanging fruit of reducing rates of urgent and emergent repair remain. For example, improving patient adherence to surveillance imaging protocols may reduce risk of TAAA rupture. More than one third of repairs were performed in response to the progressive aortic dilatation that is common in chronic distal aortic dissection; in general, these patients tend to do better than expected, and given this information, they may better prepare for the likelihood of needing additional aortic repair in the future and seek elective repair.

\section{Conflict of Interest Statement}

J.S.C. receives royalties for the 4-branched Coselli thoracoabdominal aortic graft and spoke at a sponsored meeting held by Vascutek Terumo; consults and participates in clinical research for Medtronic, Inc, Vascutek Terumo, Edwards Lifesciences, and WL Gore \& Associates; and participates in clinical research for GlaxoSmithKline and Cook, Inc. S.A.L. serves as an investigator in clinical research for Vascutek Terumo; serves as a co-investigator in clinical research for Medtronic, Inc, WL Gore \& Associates, Cook, Inc, and GlaxoSmithKline; has served as a consultant with Medtronic PS Medical, Inc; and has served on Advisory Boards for Baxter Health Care. O.P. 
consults with Medtronic, Inc, and has received travel fees from WL Gore \& Associates and Cook, Inc. T.K.R. serves as an investigator in clinical research for Edwards Lifesciences. All other authors have nothing to disclose with regard to commercial support.

You can watch a Webcast of this AATS meeting presentation by going to: http://webcast.aats.org/2015/Video/ Monday/04-27-15_4E_0730_Coselli.mp4.

The authors thank Laurie Fondren for managing subject enrollment; Ucha Comfort Elendu, Jonathan A. Hash, Arin C. Robertson, MPH, D'Arcy Wainwright, Samantha Zarda, MS, Michael Hughes, Jeffrey Whorton, and Ameena Manzoor for data collection; Stacey A. Carter, MD, for assistance with database development; Scott A. Weldon, MA, CMI, and Hiruni S. Amarasekara, MS, for creating illustrations and figures; and Stephen N. Palmer, PhD, ELS, of the Texas Heart Institute, for providing editorial support.

\section{References}

1. DeBakey ME, Cooley DA. Successful resection of aneurysm of thoracic aorta and replacement by graft. JAMA. 1953;152:673-6.

2. Etheredge SN, Yee J, Smith JV, Schonberger S, Goldman MJ. Successful resection of a large aneurysm of the upper abdominal aorta and replacement with homograft. Surgery. 1955;38:1071-81.

3. Rob C. The surgery of the abdominal aorta and its major branches. Ann R Coll Surg Engl. 1955;17:307-18.

4. DeBakey ME, Crawford ES, Garrett HE, Beall AC Jr, Howell JF. Surgical considerations in the treatment of aneurysms of the thoraco-abdominal aorta. Ann Surg. 1965;162:650-62.

5. Crawford ES. Thoraco-abdominal and abdominal aortic aneurysms involving renal, superior mesenteric, celiac arteries. Ann Surg. 1974;179:763-72.

6. Crawford ES, Crawford JL, Safi HJ, Coselli JS, Hess KR, Brooks B, et al. Thoracoabdominal aortic aneurysms: preoperative and intraoperative factors determining immediate and long-term results of operations in 605 patients. $J$ Vasc Surg. 1986;3:389-404.

7. Svensson LG, Crawford ES, Hess KR, Coselli JS, Safi HJ. Experience with 1509 patients undergoing thoracoabdominal aortic operations. J Vasc Surg. 1993;17: 357-70.

8. Overman DM, Jacobs JP, Prager RL, Wright CD, Clarke DR, Pasquali SK, et al. Report from the Society of Thoracic Surgeons National Database Workforce: clarifying the definition of operative mortality. World J Pediatr Congenit Heart Surg. 2013;4:10-2.

9. LeMaire SA, Miller CC III, Conklin LD, Schmittling ZC, Köksoy C, Coselli JS. A new predictive model for adverse outcomes after elective thoracoabdominal aortic aneurysm repair. Ann Thorac Surg. 2001;71:1233-8.

10. Wong DR, Parenti JL, Green SY, Chowdhary V, Liao JM, Zarda S, et al. Open repair of thoracoabdominal aortic aneurysm in the modern surgical era: contemporary outcomes in 509 patients. J Am Coll Surg. 2011;212:569-81.

11. LeMaire SA, Price MD, Green SY, Zarda S, Coselli JS. Results of open thoracoabdominal aortic aneurysm repair. Ann Cardiothorac Surg. 2012;1:286-92.

12. LeMaire SA, Carter SA, Volguina IV, Laux AT, Milewicz DM, Borsato GW, et al. Spectrum of aortic operations in 300 patients with confirmed or suspected Marfan syndrome. Ann Thorac Surg. 2006;81:2063-78.

13. Coselli JS, Bozinovski J, LeMaire SA. Open surgical repair of 2286 thoracoabdominal aortic aneurysms. Ann Thorac Surg. 2007;83:S862-4; discussion S90-2.

14. Coselli JS, LeMaire SA, Weldon SA. Extent II repair of thoracoabdominal aortic aneurysm secondary to chronic dissection. Ann Cardiothorac Surg. 2012;1: 394-7.

15. de la Cruz KI, LeMaire SA, Weldon SA, Coselli JS. Thoracoabdominal aortic aneurysm repair with a branched graft. Ann Cardiothorac Surg. 2012;1:381-93.

16. Coselli JS, de la Cruz KI, Preventza O, LeMaire SA, Weldon S. Extent II thoracoabdominal aortic aneurysm repair: how I do it. Semin Thorac Cardiovasc Surg. [In press].
17. Coselli JS, LeMaire SA. Left heart bypass reduces paraplegia rates after thoracoabdominal aortic aneurysm repair. Ann Thorac Surg. 1999;67:1931-4.

18. Coselli JS, LeMaire SA, Köksoy C, Schmittling ZC, Curling PE. Cerebrospinal fluid drainage reduces paraplegia after thoracoabdominal aortic aneurysm repair: results of a randomized clinical trial. J Vasc Surg. 2002;35:631-9.

19. Köksoy C, LeMaire SA, Curling PE, Raskin SA, Schmittling ZC, Conklin LD, et al. Renal perfusion during thoracoabdominal aortic operations: cold crystalloid is superior to normothermic blood. Ann Thorac Surg. 2002; 73:730-8

20. LeMaire SA, Jamison AL, Carter SA, Wen S, Alankar S, Coselli JS. Deployment of balloon expandable stents during open repair of thoracoabdominal aortic aneurysms: a new strategy for managing renal and mesenteric artery lesions. Eur J Cardiothorac Surg. 2004;26:599-607.

21. MacArthur RG, Carter SA, Coselli JS, LeMaire SA. Organ protection during thoracoabdominal aortic surgery: rationale for a multimodality approach. Semin Cardiothorac Vasc Anesth. 2005;9:143-9.

22. LeMaire SA, Jones MM, Conklin LD, Carter SA, Criddell MD, Wang XL, et al. Randomized comparison of cold blood and cold crystalloid renal perfusion for renal protection during thoracoabdominal aortic aneurysm repair. J Vasc Surg. 2009;49:11-9.

23. Johansen KL, Chertow GM. Chronic kidney disease mineral bone disorder and health-related quality of life among incident end-stage renal-disease patients. $J$ Ren Nutr. 2007;17:305-13.

24. Wijeysundera DN, Karkouti K, Beattie WS, Rao V, Ivanov J. Improving the identification of patients at risk of postoperative renal failure after cardiac surgery. Anesthesiology. 2006;104:65-72.

25. Aftab M, Songdechakraiwut T, Green SY, Zarda S, Price MD, Nalty CC, et al. Contemporary outcomes of open thoracoabdominal aortic aneurysm repair in octogenarians. J Thorac Cardiovasc Surg. 2015;149:S134-41.

26. Juvonen T, Ergin MA, Galla JD, Lansman SL, Nguyen KH, McCullough JN, et al. Prospective study of the natural history of thoracic aortic aneurysms. Ann Thorac Surg. 1997;63:1533-45.

27. Conrad MF, Crawford RS, Davison JK, Cambria RP. Thoracoabdominal aneurysm repair: a 20-year perspective. Ann Thorac Surg. 2007;83:S856-61.

28. Chiesa R, Melissano G, Civilini E, de Moura ML, Carozzo A, Zangrillo A. Ten years experience of thoracic and thoracoabdominal aortic aneurysm surgical repair: lessons learned. Ann Vasc Surg. 2004;18:514-20.

29. Lombardi JV, Carpenter JP, Pochettino A, Sonnad SS, Bavaria JE. Thoracoabdominal aortic aneurysm repair after prior aortic surgery. J Vasc Surg. 2003;38: 1185-90.

30. Patel VI, Ergul E, Conrad MF, Cambria M, LaMuraglia GM, Kwolek CJ, et al. Continued favorable results with open surgical repair of type IV thoracoabdominal aortic aneurysms. J Vasc Surg. 2011;53:1492-8.

31. Kulik A, Castner CF, Kouchoukos NT. Outcomes after thoracoabdominal aortic aneurysm repair with hypothermic circulatory arrest. J Thorac Cardiovasc Surg. 2011;141:953-60.

32. Zoli S, Roder F, Etz CD, Brenner RM, Bodian CA, Lin HM, et al. Predicting the risk of paraplegia after thoracic and thoracoabdominal aneurysm repair. Ann Thorac Surg. 2010;90:1237-45.

33. Coselli JS, LeMaire SA, Conklin LD, Köksoy C, Schmittling ZC. Morbidity and mortality after extent II thoracoabdominal aortic aneurysm repair. Ann Thorac Surg. 2002;73:1107-16.

34. Omura A, Tanaka A, Miyahara S, Sakamoto T, Nomura Y, Inoue T, et al. Early and late results of graft replacement for dissecting aneurysm of thoracoabdominal aorta in patients with Marfan syndrome. Ann Thorac Surg. 2012;94:759-65.

35. Mommertz G, Sigala F, Langer S, Koeppel TA, Mess WH, Schurink GW, et al. Thoracoabdominal aortic aneurysm repair in patients with Marfan syndrome. Eur J Vasc Endovasc Surg. 2008;35:181-6.

36. Coselli JS, Green SY, Zarda S, Nalty CC, Price MD, Hughes MS, et al. Outcomes of open distal aortic aneurysm repair in patients with chronic DeBakey type I dissection. J Thorac Cardiovasc Surg. 2014;148:2986-93. e1-2.

37. Dayama A, Sugano D, Reeves JG, Rivera A, Tsilimparis N. Early outcomes and perioperative risk assessment in elective open thoracoabdominal aortic aneurysm repair: an analysis of national data over a five-year period. Vascular. 2016; 24:3-8.

38. Sagban T, Kocabas F, Balzer K, Duran M, Weis-Müller B, Grabitz K, et al. Outcome of septuagenarians and octogenarians after open TAAA repair. Ital J Vasc Endovasc Surg. 2012;19:21-30.

39. Tanaka H, Minatoya K, Matsuda H, Sasaki H, Iba Y, Oda T, et al. Embolism is emerging as a major cause of spinal cord injury after descending 
and thoracoabdominal aortic repair with a contemporary approach: magnetic resonance findings of spinal cord injury. Interact Cardiovasc Thorac Surg. 2014;19:205-10.

40. Zangrillo A, Buratti L, Carozzo A, Casiraghi G, Landoni G, Lembo R, et al. Intrathecal lactate as a predictor of early- but not late-onset spinal cord injury in thoracoabdominal aneurysmectomy. J Cardiothorac Vasc Anesth. 2014;28: 473-8.

41. Bensley RP, Curran T, Hurks R, Lo RC, Wyers MC, Hamdan AD, et al. Open repair of intact thoracoabdominal aortic aneurysms in the American College of Surgeons National Surgical Quality Improvement Program. J Vasc Surg. 2013; 58:894-900.

42. Jacobs MJ, de Mol BA, Elenbaas T, Mess WH, Kalkman CJ, Schurink GW, et al. Spinal cord blood supply in patients with thoracoabdominal aortic aneurysms. $J$ Vasc Surg. 2002;35:30-7.

43. Lancaster RT, Conrad MF, Patel VI, Cambria MR, Ergul EA, Cambria RP. Further experience with distal aortic perfusion and motor-evoked potential monitoring in the management of extent I-III thoracoabdominal aortic aneurysms. J Vasc Surg. 2013;58:283-90.

44. Kouchoukos NT, Kulik A, Castner CF. Outcomes after thoracoabdominal aortic aneurysm repair using hypothermic circulatory arrest. J Thorac Cardiovasc Surg. 2013;145:S139-41.

45. Di Luozzo G, Geisbusch S, Lin HM, Bischoff MS, Schray D, Pawale A, et al. Open repair of descending and thoracoabdominal aortic aneurysms and dissections in patients aged younger than 60 years: superior to endovascular repair? Ann Thorac Surg. 2013:95:12-9.

46. Estrera AL, Sandhu HK, Charlton-Ouw KM, Afifi RO, Azizzadeh A, Miller CC III, et al. A quarter century of organ protection in open thoracoabdominal repair. Ann Surg. 2015;262:660-8.

47. Coselli JS, Bozinovski J, Cheung C. Hypothermic circulatory arrest: safety and efficacy in the operative treatment of descending and thoracoabdominal aortic aneurysms. Ann Thorac Surg. 2008;85:956-64.

48. Dias NV, Sonesson B, Kristmundsson T, Holm H, Resch T. Short-term outcome of spinal cord ischemia after endovascular repair of thoracoabdominal aortic aneurysms. Eur J Vasc Endovasc Surg. 2015;49:403-9.

49. Bisdas T, Panuccio G, Sugimoto M, Torsello G, Austermann M. Risk factors for spinal cord ischemia after endovascular repair of thoracoabdominal aortic aneurysms. J Vasc Surg. 2015;61:1408-16.

50. Etz CD, Weigang E, Hartert M, Lonn L, Mestres CA, Di Bartolomeo R, et al. Contemporary spinal cord protection during thoracic and thoracoabdominal aortic surgery and endovascular aortic repair: a position paper of the vascular domain of the European Association for Cardio-Thoracic Surgery. Eur J Cardiothorac Surg. 2015;47:943-57.

Key Words: aneurysm (aorta), aortic dissection, aortic operation, outcomes, thoracoabdominal

\section{Discussion}

Dr N. Kouchoukos (St Louis, Mo). Dr Coselli and colleagues have presented their truly monumental experience with open thoracoabdominal aneurysm repair, more than 3000 procedures in an approximately 30-year span. This is the largest series of surgically treated patients ever reported, and it is unlikely that an experience of this magnitude will ever be duplicated. Those of us who perform or have observed thoracoabdominal aneurysm repairs recognize that the intense effort, both physical and intellectual, and the teamwork and man-hours required to achieve the excellent outcomes as he has reported are truly extraordinary.

Dr Coselli has based his experience on the pioneering work of his distinguished predecessors at Baylor College of Medicine, Drs Michael DeBakey, Denton Cooley, and Stanley Crawford, who were among the first to perform these procedures and who established a center for the treatment of aortic disease that is world renowned and remains at the forefront. I am certain that Stanley Crawford, wherever he is, is smiling and extremely proud of what Dr Coselli has accomplished to advance the treatment of thoracoabdominal aortic disease.

The article, as you saw, contains an enormous amount of extremely important and rigorously analyzed data that could not be adequately presented in the time allotted. I will limit my remarks and questions to a few specific areas.

Dr Coselli, did you observe a reduction in mortality and in the prevalence of major complications over time as you modified your technique? This was not directly addressed in the article because the variable of year of operation did not appear in the multivariate analysis.

Dr Coselli. Dr Kouchoukos, your efforts have been at the forefront of TAAA repair for many years. We did a rigorous analysis by various eras. We looked at all of the major morbidities and mortality, and I will have to say that even with controlling for increased risks of patient comorbidity over time, the actual results did not significantly change from our earliest era to our most contemporary era. Of note, we did identify brief time periods in which outcomes improved, such as several periods when we had no cases of paraplegia for more than a year.

Dr Kouchoukos. You observed in your study and others, including our group, have observed that the Crawford Extent II and III procedures are associated with the highest early mortality and prevalence of major complications, most important, permanent paralysis. Do you believe that the risks of spinal cord ischemic injury could be reduced by the addition of other interventions, such as monitoring of evoked potentials, deeper hypothermia, pharmacologic agents, and avoidance of sequential clamping to reduce the risk of embolization, or have we reached an irreducible minimum risk for this complication?

Dr Coselli. We report solely our own experience, and thus, can only speculate on what altering that experience might derive. I would say this, that in the earlier years, and particularly with Stanley Crawford, our greatest concerns were in the Extent II category and cases of aortic dissection. But with cerebrospinal fluid drainage and left heart bypass, having chronic dissection has become beneficial from a risk standpoint in Extent II repairs. Not so with the Extent IIIs and Extent IVs, which over the years have evolved into a group of patients with more atherosclerosis and less dissection, older age, more comorbidities, particularly pulmonary and renal preoperatively, and a heavy layer of superimposed atherosclerosis. This group has actually become more challenging.

We have operated in approximately 50 patients with deep HCA. In our own experience with those cases 
being patients in whom it wasn't the primary strategy but one we thought necessary because cross-clamping was not possible secondary to anatomy and other complicating factors. It is very likely that in our cohort of patients with deep HCA, the results are similar to the overall group, if you adjust for the inherent risks present in this highly select cohort of patients with many complicating factors.

Dr Kouchoukos. Do you think we are at the irreducible minimum? Are we ever going to further reduce the risk of paraplegia?

Dr Coselli. Although there remains potential to further reduce the risk of postoperative paraplegia in the future, I think we are a long way off from bringing it to zero.

Dr Kouchoukos. Finally and perhaps most important, how do you see the role of open thoracoabdominal aneurysm repair in the next 15 to 30 years? Will endovascular repair supplant open repair as the preferred method of management or will open repair still have a role?

Dr Coselli. I think open repair is going to be around for some time. There remains a concern with some of the engineering and technologic issues present in purely endovascular repair of the thoracoabdominal aneurysm, especially for extent II repairs. Because of this, I think this supplantation is still in the future. However, a lot of work is being done by a number of institutions and endovascular companies to do just that. On the open side of TAAA repair, we have developed a new indication for operation, and that is the failed endovascular repair; somewhat unpredictably, we have seen rapid expansion of the aneurysm and rupture of patients with endovascular stent-grafts in place, and they have to come back and be rescued in an open fashion.

Dr L. Svensson (Cleveland, Ohio). I congratulate you, Joe, on an incredible series, and thus you have shown that you have taken over the mantle of both Dr DeBakey and Dr Crawford, and, as Dr Kouchoukos said, I don't think this will ever be equaled again. By my back of the envelope calculation, the time you spent on this series is some 15,000 hours.

The challenging group is obviously the type IIs, and that's where we all see the complications. I was wondering, apart from the role of stenting in the type Is and type IVs, what do you think we should be doing for the IIs? Maybe cooling the patients more, being more aggressive in prescreening them for the intercostal or lumbar arteries?

Dr Coselli. Basically, I agree entirely with everything you have said. There is some potential for intercostal and anterior spinal artery identification with emerging technology and software that may provide substantial advantages regarding this identification in a few years. Although for now, purely eTAAA repair is clearly directed at the low-hanging fruit, such as the extent IV repairs and probably the extent I repairs. However, we are going to have to be very careful in choosing a method of repair for the extent II category, particularly for younger patients and those with connective tissue disorders or chronic dissection, who generally do well with open repair. 